\title{
An elasto-plastic self-consistent model with hardening based on dislocation density, twinning and de-twinning: Application to strain path changes in HCP metals
}

\author{
Milovan Zecevic ${ }^{\mathrm{a}}$, Marko Knezevic ${ }^{\mathrm{a},{ }^{*}}$, Irene J. Beyerlein ${ }^{\mathrm{b}}$, Carlos N. Toméc \\ ${ }^{a}$ Department of Mechanical Engineering, University of New Hampshire, Durham, NH 03824, USA \\ ${ }^{\mathrm{b}}$ Theoretical Division, Los Alamos National Laboratory, Los Alamos, NM 87545, USA \\ ${ }^{\mathrm{c}}$ Materials Science and Technology Division, Los Alamos National Laboratory, Los Alamos, NM 87545, USA
}

\begin{abstract}
In this work, we develop a polycrystal mean-field constitutive model based on an elastic-plastic self-consistent (EPSC) framework. In this model, we incorporate recently developed subgrain models for dislocation density evolution with thermally activated slip, twin activation via statistical stress fluctuations, reoriented twin domains within the grain and associated stress relaxation, twin boundary hardening, and de-twinning. The model is applied to a systematic set of strain path change tests on pure beryllium (Be). Under the applied deformation conditions, Be deforms by multiple slip modes and deformation twinning and thereby provides a challenging test for model validation. With a single set of material parameters, determined using the flowstress vs. strain responses during monotonic testing, the model predicts well the evolution of texture, lattice strains, and twinning. With further analysis, we demonstrate the significant influence of internal residual stresses on: 1) the flow stress drop when reloading from one path to another, 2) deformation twin activation, 3) de-twinning during a reversal strain path change, and 4) the formation of additional twin variants during a cross-loading sequence. The model presented here can, in principle, be applied to other metals, deforming by multiple slip and twinning modes under a wide range of temperature, strain rate, and strain path conditions.
\end{abstract}

Keywords: Hexagonal metals; Polycrystals; Dislocations; Twinning; De-twinning; Texture

\footnotetext{
${ }^{*}$ Corresponding author at: University of New Hampshire, Department of Mechanical Engineering, 33 Academic Way, Kingsbury Hall, W119, Durham, New Hampshire 03824, United States. Tel.: 603862 5179; fax: 603862 1865; email: marko.knezevic@unh.edu
} 


\subsection{Introduction}

Under an applied mechanical strain, hexagonal-close packed (HCP) polycrystalline metals, such as $\mathrm{Mg}$, $\mathrm{Ti}, \mathrm{Be}$, and $\mathrm{Zr}$, can deform via combinations of slip and twinning, even in ambient conditions [1, 2]. Under strain path changes the relative activities of slip and twinning can change and in particular, new slip and twin variants can activate within the same grain, former slip can reverse direction, and twin domains can shrink (de-twinning). These basic processes are microscopic, occurring in the interior of the grain and across or in the vicinity of the grain boundaries. The relative proportions of slip and twinning accommodating plastic strain and when and where they activate in the grain structure during a strain path have a profound effect on the flow stress-strain response and microstructure evolution.

A number of material models have been developed to relate such details of slip and twinning in HCP metals to the macroscopic response. They range from analytical models [3] to polycrystalline upper-bound schemes such as Taylor $[4,5]$ and mean-field self-consistent models $[6,7]$ to spatially resolved techniques, such as crystal plasticity finite element [8-11] and Green's function fast Fourier transform [12] models. While the latter class of models can be used for detailed simulations of grain-grain interactions and stress and strain localizations [10, 13-15], the mean-field constitutive models are more computationally efficient and have proven effective for predicting the average flow stress and texture evolution of grain aggregates over moderate to large strains [6, 7, 16-19]. Another advantage lies in the schemes they use for deformation twinning and linking its micromechanics to behavior of the polycrystal [20-23]. However explicit incorporation of deformation twinning in spatially resolved techniques is meeting challenges and is the subject of ongoing model development in the field [24].

In some cases, mean-field polycrystal models have served as the fundamental constitutive model within a larger finite element simulation. The polycrystal model provides the stress-strain relationship of a local material volume or material element undergoing homogeneous deformation of a finite element simulation and the FE simulation treats the variation in deformation from point to point across the sample. Such multi-scale model approaches have been used for simulations of compression and tension tests [25], bending [26-28], rolling [29], cupdrawing [30, 31], sheet hydroforming [32], tube extrusion [33], and bulk forming [34]. As these studies show, when a polycrystal model is used as the material point constitutive response in 
place of the commonly used empirical or phenomenological laws, then heterogeneity in texture and other microstructural features can be predicted.

Considering their widespread use and value, it is important that the polycrystal constitutive models take into account the mechanisms and physics driving deformation operating at a microscopic scale. Over the years, polycrystal models have been developed to account for a number of subgrain level phenomena for the evolution of crystallographic slip resistance with strain. Hardening has been modeled via phenomenological laws, such as the Voce law [35] and Mechanical Threshold Stress [36, 37], or based on the evolution of stored dislocations [21, 3840], as well as both stored and mobile dislocations within the grain [41]. Representing the effects of deformation twinning in these models is commonly more demanding than slip. Deformation twins affect the microstructure in two ways: they reorient the lattice within a finite domain within a grain and they introduce a twin-matrix boundary between the original parent grain and the twin domain $[42,43]$. These effects impact dislocation processes in at least two ways. The twin-matrix boundaries can hinder slip (boundary effect) and the reoriented twin domains can provide new orientations within which the propensity for slip changes (lattice reorientation effect). Earlier analytical and polycrystalline mean-field models accounted for twinning as a pseudo-slip process [44], and considered the reorientation effect, via: (i) the predominant twin reorientation (PTR) method [45], (ii) the volume fraction transfer (VFT) scheme [46], (iii) the total Lagrangian approach [47, 48], and (iv) the composite grain (CG) method [49]. The most recent treatments take into account both effects, via the composite grain model for reorientation for a given number of twins $n$ per grain and a Hall-Petch-type model for the spacing between adjacent twin boundaries [20, 39, 50].

Yet still, the effects of twinning in stress-strain response were not captured well without substantial parameter fitting $[39,49,51]$. Some recent works suggest that the problem may lie in the fact that most of these models treated the effects of twinning after the twin had nucleated and not twin nucleation itself. For twin nucleation, deterministic rules were typically used. In actuality, twinning calls for a probabilistic treatment. When and where twins nucleated is statistical in nature due to defect variations in the microstructure and fluctuations in intergranular (type 2) and intra-granular (type 3) stresses. Most recently, probability models for twin nucleation have been introduced, leading to significant improvements in the capability to capture both the twinned microstructure and stress-strain response [20, 21, 52]. In those models, the 
characteristic threshold stress to first activate twinning was probabilistic and the stress concentrations for driving nucleation were randomly fluctuating. At nucleation, the reoriented twin lamellae were introduced and the stress and strain of the twin and matrix updated. After nucleation, the critical stresses governing the expansion of the reoriented twin domain and hence shrinkage of the matrix domain were modeled deterministically. The above effects of probabilistic activation of deformation twins have thus far been implemented into a visco-plastic self-consistent (VPSC) formulation, which only accounts for stresses derived from plastic strains and ignors elastic residual stresses of type 2. Clearly treatments that also consider the effect of elastic strains would be more desirable.

In most of the modeling advancements reviewed above, the polycrystal constitutive laws were tested under loadings applied in one direction and fixed deformation conditions, whereas in practice, loading situations often involve changes temperatures, strain path, strain rate, etc., such as in fatigue and forming operations. To model stress-strain behavior during a sequence of two or more strain paths, each differing in load direction, temperature, or strain rate, it has been found necessary to account for additional micro-scale phenomena often missed in monotonic loading treatments, such as backstresses, substructure development, glide of special dislocations (“polarized dislocations") [53-55], and/or de-twinning [56, 57].

Many of the foregoing phenomena are driven by a complex coupling of elastic and plastic strain fields. For instance, the residual stresses and strains left in the microstructure after one strain path are expected to affect the propensity of plastic slip, deformation twinning, or detwinning in subsequent paths. However, many of the modeling attempts to date have not completely captured the combination of dislocation motion and residual elastic strain development. Previously, constitutive models based on Taylor or VPSC have employed the idea of reversible dislocations to model the flow stress during cyclic loading and strain path changes [53-55, 57-59]. While they involve sophisticated models for dislocation density evolution (as opposed to phenomenological ones) they also neglect elastic strain development. Some polycrystalline models approaches have accounted for the development of elastic strains and their effects on deformation mechanisms. Constitutive models using an elasto-plastic selfconsistent (EPSC) approach have been applied to cyclic loading cases for stainless steel [60]. Most notable is the recent development of the elasto-VPSC (E-VPSC) models and its modeling 
of de-twinning and loading and unloading sequences [61]. Yet still, the models for hardening of slip resistance were based on phenomenological functions and not based on dislocation storage.

Here, a multi-scale constitutive model for hexagonal close packed (HCP) metals is developed. It is predominantly a meso-scale model, covering the length scale range from the subgrain scale to the scale of the polycrystalline aggregate. We use EPSC [7] as it enables both elastic and plastic strains to play a role in slip, twinning, and de-twinning. To account for these deformation mechanisms, we incorporate into the EPSC formulation several subgrain and grainlevel models that have up to now only been used in combination with the VPSC formulation: dislocation density based hardening, internal stress fluctuations for twin nucleation, CG twinning model and de-twinning. We additionally advance the twinning model from VPSC to account for multiple twin domains of different variants within the same grain in EPSC. To distinguish this model from prior modeling efforts, we refer hereinafter to it as the multi-scale EPSC (MS-EPSC) model.

The standard version of the EPSC model has been used in the past for the interpretation of insitu neutron diffraction data $[60,62]$, a method to experimentally probe grain-level deformation behavior within bulk samples through measurements of internal strains. The high-penetration depth of neutrons and high-energy x-rays in most materials makes it possible to probe the internal structure within bulk samples under and after unloading. Changes in diffraction peak locations are associated with internal elastic strain development. An advantage of coupling EPSC modeling and in-situ diffraction is that the model and the experiment probe the polycrystal in similar ways allowing for successful interpretation of the diffraction results. A fraction of grains with a common orientation but a variety of grain neighborhoods gives rise to an averaged diffraction response, and the EPSC model allows determination of the average response of a similar subset of grains embedded within an averaged (effective) environment.

In this work, the MS-EPSC model is applied to a set of strain path change uniaxial tests on pure Be reported previously in [63]. During these tests, evolution of flow stress, texture, twin volume fraction, and lattice strain, were measured. All these data can potentially be predicted and interpreted by the EPSC model. Experimental measurements in [63] indicate that within this test matrix, some strain paths involve slip, some both slip and twinning, and others slip and detwinning. The aim of our application to this Be data set is two-fold: 1) to predict when slip, twinning, and de-twinning occurs and its effect on texture and stress-strain and 2) to understand 
the effect of internal residual stresses on the activation of slip, twinning and de-twinning during two different types of strain path changes, a pseudo-reversal and a pseudo-cross loading.

After characterization of the material parameters with a separate data set, the MS-EPSC model capability is demonstrated by good agreement in its predictions of evolution texture, twin fraction in twinning and de-twinning. Furthermore, as arguably the most stringent test of the model, the predictions for the evolution of residual lattice strains are shown to compare well with the data measured in situ in the high-energy x-ray diffractometer [63]. Through this effort, we reveal the significant effect of internal residual stresses on twin activation, the change in yield and flow stresses during the strain path change, and de-twinning. These findings were not possible with prior studies on HCP polycrystal behavior during strain path changes.

The paper is structured as follows. First, we present the multiscale (MS)-EPSC constitutive model, starting from a review of the EPSC scheme, including the grain-level twinning and detwinning models and ending with models for slip and twin activation at the subgrain level. The model for treating multiple twin variants within a single grain is subsequently described. We then present our application to stress, texture, and lattice strain measurements made during uniaxial strain path change tests on high-purity Be. We end with a discussion on the implications on the specific roles of internal residual stresses.

\subsection{Modeling framework}

\subsection{Polycrytalline model: Elastic-Plastic Self-Consistent (EPSC) formulation}

The EPSC model relates the deformation of individual grains to the polycrystalline aggregate response. The model follows the formulation given in Ref. [64]. Details can also be found in [7, 65]. Below we only review the essential details.

In EPSC, the polycrystal is represented by a number of grains with a given volume fraction and crystallographic orientation. Each grain is modeled as an ellipsoidal inclusion embedded in a homogeneous matrix with average properties of the aggregate polycrystal. By working in small strain increments, a linearized relation between stress and strain is employed, where the increments in the macroscopic stress and strain are:

$$
d \boldsymbol{\sigma}=\mathbf{L} d \boldsymbol{\varepsilon} .
$$

In Eq. (1), $\mathbf{L}$ is the instantaneous elasto-plastic stiffness tensor of the polycrystal matrix. The response of each grain follows from solving the stress equilibrium and compatibility relations for 
an inclusion embedded in a homogeneous anisotropic matrix under applied loads [7, 66]. Increments in polycrystal stress and strain are equal to the volume average of the grain stress increment and strain increments as:

$$
d \boldsymbol{\sigma}=\left\langle d \boldsymbol{\sigma}^{c}\right\rangle \quad \text { and } \quad d \boldsymbol{\varepsilon}=\left\langle d \boldsymbol{\varepsilon}^{c}\right\rangle .
$$

The condition that the average of the grain increments are equal to the overall polycrystal increments leads to a self-consistent equation for the overall elasto-plastic stiffness $\mathbf{L}$, which is calculated iteratively in a given increment using a standard self-consistent procedure [7]. At this point the stress states, strain states, and slip/twin resistances (through the hardening law described later) are updated incrementally for the current deformation step $n+1$, with respect to their previously converged values, noted by the superscript $n$. For example, the new stress of the polycrystalline aggregate is calculated from the overall stress increment, $d \boldsymbol{\sigma}$, associated with the strain increment, $d \boldsymbol{\varepsilon}$, as:

$$
\boldsymbol{\sigma}^{n+1}=\boldsymbol{\sigma}^{n}+d \boldsymbol{\sigma} .
$$

\subsection{Single crystal plasticity model}

In our notation, "." is a contracted or dot product and " $\otimes$ " is an uncontracted or tensor dyadic product. For the individual grains (ellipsoidal inclusions), the constitutive relation between increment in stress and increment in strain is linearized as:

$$
d \boldsymbol{\sigma}^{c}=\mathbf{L}^{c} d \boldsymbol{\varepsilon}^{c}
$$

where $\mathbf{L}^{\mathbf{c}}$ is the incremental elasto-plastic stiffness tensor (aka instantaneous moduli) in the grains and is defined as:

$$
\mathbf{L}^{c}=\mathbf{C}^{c} \cdot\left(\mathbf{I}-\sum_{s} \mathbf{m}^{s} \otimes \mathbf{f}^{s}\right)
$$

where $\mathbf{C}^{c}$ is the elastic stiffness tensor of a single crystal and $\mathbf{m}^{s}$ is the Schmid tensor for the $s^{\text {th }}$ slip system. The matrix, $\mathbf{f}^{s}$, relates an increment in shear strain on $s^{\text {th }}$ slip system to the total increment in strain, $d \gamma^{s}=\mathbf{f}^{s} d \boldsymbol{\varepsilon}^{c}$, and is defined for a given set of active systems as:

$$
\mathbf{f}^{s}=\sum_{s^{\prime}}\left(\mathbf{X}^{-1}\right)^{s s^{\prime}} \mathbf{m}^{s^{\prime}} \cdot \mathbf{C}^{c}, \mathbf{X}^{s s^{\prime}}=h^{s s^{\prime}}+\mathbf{m}^{s} \cdot \mathbf{C}^{c} \cdot \mathbf{m}^{s^{\prime}}
$$

The slip resistance on a slip system is a function of increments in shear according to: 


$$
d \tau_{c}^{s}=\sum_{s^{\prime}} h^{s s^{\prime}} d \gamma^{s^{\prime}}
$$

where $h^{s s^{\prime}}$ is hardening matrix (i.e., instantaneous hardening coefficients). It should also be

noted that $\mathbf{f}^{s}$ is dependent on the hardening law through $h^{s s^{\prime}}$. Elements of the hardening matrix are themselves a function of dislocation density. (The model for the evolution of dislocation density will be described in section 2.4.1.) It can be seen that the tensor $\mathbf{L}^{c}$ is a function of the active slip systems, elastic stiffness tensor, and hardening law. When the set of active slip and twinning systems are known, then $\mathbf{L}^{c}$ can be calculated. In order for a slip or twinning system to be active, it must satisfy two conditions: (1) $\mathbf{m}^{s} \cdot \boldsymbol{\sigma}^{c}=\tau_{c}^{s}$, meaning that the resolved shear stress on the slip system has to be equal to the slip resistance (stress needs to be on the single crystal yield surface) and (2) $\mathbf{m}^{s} \cdot d \boldsymbol{\sigma}^{c}=d \tau_{c}^{s}$, meaning that the slip/twin system has to remain on the evolving single crystal yield surface in a given increment as stress evolves due to hardening. In addition, the formulation allows for only positive shear on a given slip system, i.e., $\Delta \gamma^{s}>0$, since reverse shear of this slip system would be captured as positive slip on the opposite-signed slip system. The slip systems are defined such that the two slip systems sharing the same plane normal but having opposite sense of shear are independently considered.

\subsection{Twinning and de-twinning models}

The grains in the present MS-EPSC model can either be untwinned or twinned. A nucleation criterion, which will be described in section 2.4.3, is used to determine when and if a grain twins and via which variant. If a grain is to twin, then the grain model is converted to a composite grain (CG) model. The CG model accounts for both the changes in morphology and crystallographic reorientation associated with twinning [49, 67]. In the CG model, there are two ways in which a twinned grain has been treated. In the earlier version, a CG grain was a single laminated inclusion in which continuity of stresses and strains at the matrix-twin boundary is enforced [49]. In the most recent versions and the one used here, the CG grain was modeled as two separate inclusions that are decoupled mechanically [27, 39, 40]. At the moment of twin nucleation, the grain is transformed into a representative twin and matrix inclusion with the twinmatrix orientation relationship. For the shape of the twin and matrix inclusions, the long axis is made to lie along the twin plane and the short axis of the ellipsoids is aligned perpendicular to the twin plane. 
After nucleation, the twin inclusion is allowed to thicken. Twin thickening is governed by a separate growth model, which will be described in section 2.4.2. During thickening, volume fraction from the parent matrix inclusion is transferred to the twin inclusion. Accordingly, the parent matrix ellipsoid shrinks and twin ellipsoid thickens. Apart from the volume transfer, the twin and the parent matrix ellipsoids are treated as independent inclusions in the model. Slip and secondary twin activity within the reoriented twin domains are allowed (without allowing for reorientation of the secondary twins). Also, the initial twin-matrix orientation relationship could change during subsequent deformation.

A macroscopic strain path change usually leads to a change in the flow stress. The change depends on the strain level reached during forward loading, changes in load conditions (e.g., temperature, strain rate), and the relative orientation of the forward and reload paths. Such changes are induced not only by the subgrain structure but also by the crystallographic texture during forward loading. Twinning plays a major role in both aspects by introducing twin boundaries and by altering texture development during forward loading. Upon reloading, twins formed during forward load can resist new slip or twinning non-coplanar to the twin boundaries, can provide domains for new slip, or can de-twin. The original CG model [49] as well as all subsequent applications of the CG model do not account for the formation of multiple twin variants per grain.

As an advancement, we adopt the scheme from [62] to allow the activation of multiple twin variants per grain in the CG twinning model in EPSC. The orientation of the twin domain grains is easily determined from the crystallographic relation between the parent and corresponding twin variant. The twin domain ellipsoids for a given twin variant grow according to the activity of that twin variant. Also the volume fractions of the growing twin variants are transferred from the parent to the corresponding twin domains. The increment in the twin volume fraction on a twin variant, $t$, is proportional to the shear increment, $\Delta f^{t}=\Delta \gamma^{t} / S^{t}$ where $S^{t}$ is the characteristic twin shear, which for the tensile twins in $\mathrm{Be}$ is 0.189 [2]. The total volume fraction of the parent matrix ellipsoids and twin ellipsoids of all variants formed in a grain remains constant and equal to the initial volume fraction of the parent grain.

The model for the evolution of the de-twinning resistance is the same as that for twinning. It is assumed that the de-twinning of a given twin variant ellipsoid occurs by the activation of the same twin variant inside the twin domain. As this 'internal' twinning proceeds, instead of 
creating a separate secondary twin domain, the volume of the twin that should be occupied by this secondary twin is transferred from the original twin back to the parent. This process continues until the original twin volume has been entirely transferred back to the parent grain. At this point the ellipsoid of this twin variant disappears.

\subsection{Activation of slip and deformation twinning}

\subsubsection{Evolution of slip resistance with straining}

In the present model, the resistance to slip, $\tau_{c}^{s}$, is allowed to evolve with strain according to a model developed in [39]. This law for strain hardening $\tau_{c}^{s}$ is intended to cover hardening from small to large strain including Stage IV hardening of a metal. In brief, the resistance to slip $\tau_{c}^{s}$ is modeled at the level for a particular slip system $s$ belonging to a common slip mode $\alpha$ and is attributed to a sum of dislocation storage and the resistance from the lattice and internal microstructural boundaries (e.g., grain or twin boundaries). The rate of dislocation storage is governed by thermally activated dislocation glide and hence is dependent on strain rate and temperature. In several prior works, this model has been successfully used within the VPSC framework to a variety of metals of different crystal structures: hcp $\operatorname{Zr}$ [38, 39], Be [57, 68], and Mg [69, 70], BCC Nb [71, 72], FCC superalloy Haynes [73] and orthorhombic uranium [26, 27]. In order to incorporate it into the EPSC framework, the corresponding expression for the hardening rate $h^{s s^{\prime}}$ had to be newly derived. Thus, the basic formulation of this hardening model is only briefly reviewed but the derivation of $h^{s s^{\prime}}$ is presented in full.

In the following, the superscripts $s, s^{\prime}$ span the slip systems and the Greek superscripts $\alpha, \beta$ represent modes to which $s, s^{\prime}$ belong. The slip resistance per slip system, $\tau_{c}^{s}$, is a sum of a friction stress $\tau_{o}^{\alpha}$ (dependent on the Peierls stress and the initial content of dislocations), a barrier effect term $\tau_{o, H P}^{\alpha}$ (dependent on initial grain size), and forest and debris interaction stresses $\tau_{f o r}^{s}$ and $\tau_{d e b}^{\alpha}$ (dependent on a spatially random and ordered distribution of stored dislocations, respectively) [39, 74], i.e.,

$$
\tau^{s}=\tau_{o}^{\alpha}+\tau_{o, H P}^{\alpha}+\tau_{f o r}^{s}+\tau_{d e b}^{\alpha}
$$

where: 


$$
\tau_{f o r}^{s}=b^{\alpha} \chi \mu \sqrt{\rho_{f o r}^{s}}, \tau_{d e b}^{\alpha}=k_{d e b} \mu b^{\alpha} \sqrt{\rho_{d e b}} \log \left(\frac{1}{b^{\alpha} \sqrt{\rho_{d e b}}}\right), \tau_{o, H P}^{\alpha}=\mu H P^{\alpha} \sqrt{\frac{b^{\alpha}}{d_{g}}} .
$$

In the above equations, $b^{\alpha}$ is the magnitude of the Burgers vector, $d_{g}$ is the initial grain size, $\mu$ is the effective shear modulus, and $H P^{\alpha}$ is a Hall-Petch parameter. Note that $\tau_{o}^{\alpha}$ and $\tau_{o, H P}^{\alpha}$ define the initial slip resistance and do not evolve with strain. When there are twins present in the grain, the $\tau_{o, H P}^{s}$ term is defined per slip system, $s$, as $\tau_{H P}^{S}=\frac{f^{p t s}-f^{p t s, 0}}{f^{p t s, m a x}-f^{p t s, 0}} \mu H P^{\alpha} \sqrt{\frac{b^{\alpha}}{d_{m f p}^{s}}}$ and $d_{g}$ is replaced by the mean-free-path distance calculated using $d_{m f p}^{s}=\frac{\left(1-f^{p t s}\right) d_{c}}{\sin (\alpha)}[39,49]$. The angle $\alpha$ is the angle between slip plane and the PTS plane and $d_{c}=\frac{d_{g}}{n^{\text {lamellas }}}$ is the separation between twin lamellas calculated as the ratio between the grain size, $d_{g}$ and a specified number of lamellae, $n^{\text {lamellas }}$. The number of lamellae inside parent grains is chosen to be five. The term, $\frac{f^{p t s}-f^{p t s, 0}}{f^{p t s, m a x}-f^{p t s, 0}}$, represents the barrier factor and is based on the $f^{p t s, 0}$ and $f^{p t s, m a x}$ constants, which represent the minimum volume fraction identifying the PTS and the maximum volume fraction that twin can occupy in a given grain. The former $f^{p t s, 0}$ is taken to be 0.025 and the latter $f^{p t s, \max }$ is taken to be 1.0

According to the classic thermal activation model, the rate of evolution of the stored forest dislocation densities, $\rho_{\text {for }}^{s}$, with shear strain is [75-77]:

$$
\frac{\partial \rho_{f o r}^{s}}{\partial \gamma^{s^{\prime}}}=\frac{\partial \rho_{g e n, f o r}^{s}}{\partial \gamma^{s^{\prime}}}-\frac{\partial \rho_{r e m, f o r}^{s}}{\partial \gamma^{s^{\prime}}}=k_{1}^{\alpha} \sqrt{\rho_{f o r}^{s}}-k_{2}^{\alpha}(\dot{\varepsilon}, T) \rho_{f o r}^{s}, \quad d \rho_{f o r}^{s}=\frac{\partial \rho_{f o r}^{s}}{\partial \gamma^{s^{\prime}}}\left|d \gamma^{s^{\prime}}\right|
$$

where $k_{1}^{\alpha}$ is an adjustable coefficient for dislocation storage by statistical trapping of gliding dislocations by forest obstacles and $k_{2}^{\alpha}$ is a rate-sensitive coefficient that defines dynamic recovery by thermally activated mechanisms. The latter coefficient, $k_{2}^{\alpha}$, is given by [39]:

$$
\frac{k_{2}^{\alpha}}{k_{1}^{\alpha}}=\frac{\chi b^{\alpha}}{g^{\alpha}}\left(1-\frac{k T}{D^{\alpha} b^{3}} \ln \left(\frac{\dot{\varepsilon}}{\dot{\varepsilon}_{o}}\right)\right)
$$

where, $k, \dot{\varepsilon}_{o}, g^{\alpha}$, and $D^{\alpha}$ are the Boltzmann constant, a reference strain rate, an effective activation enthalpy and a drag stress, respectively. Dynamic recovery is often associated with thermal activation of dislocation cross-slip and climb, and the formation of dislocation debris is 
concomitant with these recovery processes. As a consequence, in the model, the rate of debris development is coupled to the rate of recovery of all active dislocations through:

$$
d \rho_{d e b}=\sum_{\alpha} q^{\alpha} b^{\alpha} \frac{\partial \rho_{r e m, f o r}^{s}}{\partial \gamma^{s^{\prime}}}\left|d \gamma^{s^{\prime}}\right|
$$

where $q^{\alpha}$ is a dislocation recovery rate coefficient defining the fraction of an $\alpha$-type dislocations that do not annihilate but become debris.

Based on the Eq. (7), the evolution of the resistance on a slip system is done via instantaneous hardening coefficients $h^{s s^{\prime}}$. Current slip resistance, $\tau_{c}^{s}$ on a slip system $s$ is a function of dislocation densities $\left(\rho_{\text {for }}^{s}\right.$ and $\left.\rho_{\text {deb }}\right)$, which are in turn the function of the shearing strain on slip systems inside crystal $\left(\gamma^{s}\right)$. This implies that we must evaluate:

$$
h^{s s^{\prime}}=\frac{\partial \tau_{c}^{s}}{\partial \gamma^{s^{\prime}}},
$$

which is:

$$
\frac{\partial \tau_{c}^{s}}{\partial \gamma^{s^{\prime}}}=\frac{\partial \tau_{o}^{\alpha}}{\partial \gamma^{s^{\prime}}}+\frac{\partial \tau_{f o r}^{s}}{\partial \gamma^{s^{\prime}}}+\frac{\partial \tau_{d e b}^{\alpha}}{\partial \gamma^{s^{\prime}}}+\frac{\partial \tau_{o, H P}^{\alpha}}{\partial \gamma^{s^{\prime}}}
$$

Using the chain rule and based on the above equation, individual terms from equation (14) evaluate to:

$$
\begin{aligned}
& \frac{\partial \tau_{o}^{\alpha}}{\partial \gamma^{s^{\prime}}}=\frac{\partial \tau_{o, H P}^{\alpha}}{\partial \gamma^{s^{\prime}}}=0, \\
& \frac{\partial \tau_{f o r}^{s}}{\partial \gamma^{s^{\prime}}}=\frac{\partial \tau_{f o r}^{s}}{\partial \rho^{s^{\prime}}} \frac{\partial \rho^{s^{\prime}}}{\partial \gamma^{s^{\prime}}}=b^{\alpha} \chi \mu \frac{1}{2 \sqrt{\rho^{s}}} \frac{\partial \rho^{s^{\prime}}}{\partial \gamma^{s^{\prime}}}, \text { if } s=s^{\prime} \text { else } \frac{\partial \tau_{f o r}^{s}}{\partial \gamma^{s^{\prime}}}=0, \\
& \frac{\partial \tau_{d e b}^{\alpha}}{\partial \gamma^{s^{\prime}}}=\frac{\partial \tau_{d e b}^{\alpha}}{\partial \rho_{d e b}} \frac{\partial \rho_{d e b}}{\partial \gamma^{s^{\prime}}}=-k_{d e b} \mu b^{\alpha}\left[\log \left(b^{\alpha} \sqrt{\rho_{d e b}}\right)+1\right] \frac{1}{2 \sqrt{\rho_{d e b}}} \frac{\partial \rho_{d e b}}{\partial \gamma^{s^{\prime}}} .
\end{aligned}
$$

When twins are present in a given grain, the derivative of Hall-Petch like term with respect to shear strain on PTS inside parent enters the hardening matrix as:

$$
\begin{aligned}
& \frac{\partial \tau_{H P}^{s}}{\partial \gamma^{t, p t s}}=\frac{\partial \tau_{H P}^{s}}{\partial f^{p t s}} \frac{\partial f^{p t s}}{\partial \gamma^{t, p t s}}= \\
& \left(\frac{1}{f^{p t s, m a x}-f^{p t s, 0}} \mu H P^{\alpha} \sqrt{\frac{b^{\alpha}}{d_{m f p}^{s}}}+\frac{1}{2} \frac{f^{p t s}-f^{p t s, 0}}{f^{p t s, m a x}-f^{p t s, 0}} \mu H P^{\alpha} \sqrt{b^{\alpha}} \frac{1}{\sqrt[3]{\left(d_{m f p}^{s}\right)^{2}}} \frac{d_{c}}{\sin (\alpha)}\right) \frac{\partial f^{p t s}}{\partial \gamma^{t, p t s}}
\end{aligned}
$$


$d f^{p t s}=\left(1-\sum f^{t}\right) \frac{d \gamma^{t, p t s}}{s}$

\subsubsection{Activation and propagation of deformation twinning and de-twinning}

In the model, the nucleation and propagation of deformation twinning in parent grains are treated separately from slip. The resistance to twin activation and propagation of a twin variant, $t$, is defined as [39]:

$$
\tau_{c}^{t}=\tau_{o}^{\beta}+\tau_{o, H P}^{t}+\tau_{s l i p}^{t}
$$

Here, $\tau_{o}^{\beta}$ is a temperature-independent friction term, $\tau_{o, H P}^{\beta}$ is the Hall-Petch term for grain size effects (being inversely proportional to $d_{g}$ ) as $\tau_{o, H P}^{t}=\frac{H P^{\beta}}{\sqrt{d_{g}}}$ and for grains containing twins (inversely proportional to $d_{m f p}^{t}$ ) as $\tau_{H P}^{t}=\frac{H P^{\beta}}{\sqrt{d_{m f p}^{t}}}$ where, $d_{m f p}^{t}$ is still defined as $d_{m f p}^{t}=$ $\frac{\left(1-f^{p t s}\right) d_{c}}{\sin (\alpha)} . \tau_{\text {slip }}^{t}$ is a rate-dependent latent hardening term coupling the slip and twin/de-twin systems. The term is defined via:

$$
\tau_{\text {slip }}^{t}=\mu \sum_{j} C^{\beta \alpha}(\dot{\varepsilon}) b^{\beta} b^{\alpha} \rho_{\text {for }}^{s},
$$

where $C^{\beta \alpha}$ is the latent hardening matrix. This component of the propagation resistance accounts for hardening of all twin systems due to interaction with slip systems. Evaluating the derivatives we get:

$$
\frac{\partial \tau_{s l i p}^{t}}{\partial \gamma^{s^{\prime}}}=\frac{\partial \tau_{\text {slip }}^{t}}{\partial \rho_{\text {for }}^{s^{\prime}}} \frac{\partial \rho_{\text {for }}^{s^{\prime}}}{\partial \gamma^{s^{\prime}}}=\mu \sum_{s^{\prime}} C^{\beta \alpha} b^{\beta} b^{\alpha} \frac{\partial \rho_{f o r}^{s^{\prime}}}{\partial \gamma^{s^{\prime}}}
$$

Inside twins, the Hall-Patch like contribution to the hardening is defined in a similar way as in the parent grains. The differences are only in the calculation of the mean free path, which is now calculated using $d_{m f p}^{s}=\frac{f^{p t s} d_{c}}{\sin (\alpha)}$ and the barrier factor is taken to be unity. The Hall-Petch like term inside twins does not contribute to the hardening matrix because it does not depend on the shearing inside twins, but only on the shearing in parent grains. 
During de-twinning, the Hall-Petch like term has the opposite effect on hardening as it did during twinning; that is, while its hardening effect in the parent grains decrease, it increases in twins.

\subsubsection{Description of nucleation criteria for twinning}

Twin nucleation is assumed to take place through fluctuations in stress concentration following the framework of [20]. To include this effect in a manner similar to [20], we introduce a fluctuating stress tensor $\boldsymbol{\sigma}^{c, \text { fluct }}$ into the criterion for activating a twin system $t$. We first define a local resolved shear stress on twin system $t$ via:

$$
\mathbf{m}^{t} \cdot\left(\boldsymbol{\sigma}^{c}+\boldsymbol{\sigma}^{c, \text { fluct }}\right)=\tau^{t}
$$

Components of $\boldsymbol{\sigma}^{\text {c,fluct }}$ are sampled from normal distribution, which was found in their work to represent well the stress fluctuations calculated from a spatially resolved FFT technique. Unlike in [20], where $\boldsymbol{\sigma}^{\text {c, fluct }}$ is sampled from a multivariate normal probability distribution model, we sample stress components from a one-dimensional normal distribution with a zero mean and standard deviation defined as a function of equivalent stress of polycrystal $\left(\sigma_{e q} / 10\right.$ for normal and $\sigma_{e q} / 25$ for shear stress components).

As soon as a twin variant is tagged to 'nucleate' within a parent, $\boldsymbol{\sigma}^{\text {c, fluct }}$ no longer has an effect. The growth of this twin domain is governed by hardening law (Eq. 16), with no further contribution from $\boldsymbol{\sigma}^{\text {c,fluct }}$ and the stress state within the twin domain is calculated in the EPSC$\mathrm{CG}$ formulation as described in section 2.3. There is, however, one exception to the stress calculation and it is only applied when a twin domain ellipsoid is first formed. A freshly formed twin domain is given an initial volume fraction of $1 \%$ (called "finite initial fraction" (FIF) by [62]. In this scheme, the stress in this initial twin domain is set to the difference between a correcting stress and the parent stress. The former is the elastic deformation needed to cancel the excess plastic shear caused by formation of a twin with $1 \%$ twin fraction. The $1 \%$ value is arbitrary and we found that such a tiny value was important so that it had negligible impact on the residual lattice strains and the stress relaxation in the parent grains.

\subsection{Review of experimental work}


In prior work, pure Be was tested in situ on the 1-ID-C beamline at the Advance Photon Source, Argonne National Lab and ex situ by INSTRON in the Spectrometer for Materials Research at Temperature and Stress (SMARTS) [78] at the Lujan Center, LANSCE, Los Alamos National Laboratory under uniaxial compression at room temperature [63]. The experiment was designed to study the interactions between slip and twinning, and the mechanisms underlying Bauschinger effects and de-twinning. For experimental details, we refer the reader to [63].

The as-received Be was a rolled sheet and compression cubes were cut from it for mechanical testing. The starting material had a sharp texture with a majority of the basal poles aligned in the thru-thickness (TT) direction of the original sheet material (0001)\|TT, as is shown in Fig. 2. The uniaxial loading directions and strain rates were selected in order to alter the slip and twinning systems activated. The strain rates were $0.001 / \mathrm{s}, 1 / \mathrm{s}$ and $5 / \mathrm{s}$ and the loading direction either TT (along most of the basal poles) or in-plane, IP (normal to most of the basal poles). In IP compression, a majority of the crystals in the starting material are ideally suited for twinning and prismatic slip although basal slip is the easier slip mode. In TT compression, however, most of the crystals are well suited for pyramidal slip. Basal slip is likely in both load directions within those starting crystal orientations that deviate from (0001)||TT.

\subsection{Characterization of model parameters}

The main deformation mechanisms that occur at room temperature in pure Be have been reported in [79-81]. The easiest slip systems are the basal slip systems $\{0002\}\langle 11 \overline{2} 0\rangle$. The prismatic slip systems $\{10 \overline{1} 0\}\langle 11 \overline{2} 0\rangle$ can also occur but require higher activation stresses. Pyramidal $\{10 \overline{1} 1\}\langle 11 \overline{2} 3\rangle$ slip systems are harder than both basal and prismatic slip. Deformation normal to the basal plane can be accommodated by pyramidal slip as well as $\{10 \overline{1} 2\}\langle 10 \overline{1} 1\rangle$ deformation twinning $[42,43,82]$, which is the most common twin among hep metals. It is referred to as an extension or tensile twin since when activated, it extends the crystal in the direction of the parent basal. This twin mode re-reorients the basal pole by $84.4^{\circ}$ from being perpendicular with to being nearly aligned with the compression direction. Consequently, its activity can be detected from texture measurements [68]. Based on the above, we make available to each grain the ability to deform via prismatic slip $(\alpha=1)$, basal slip $(\alpha=2)$, pyramidal slip $(\alpha$ 
$=3$ ), and $\{1012\}$ tensile twinning $(\beta=1)$. The single-crystal elastic constants used for Be were: $C_{11}=292.3 \mathrm{GPa}, C_{12}=26.7 \mathrm{GPa}, C_{13}=14.0 \mathrm{GPa}, C_{33}=336.4 \mathrm{GPa}$ and $C_{44}=162.5 \mathrm{GPa}$ [83].

Figure 1 shows the true-stress vs plastic strain curves up to 0.22 strain and Fig. 2 displays the deformed texture to a true strain of 0.22 in IP1 compression under a strain rate of 5/s, measured by neutron diffraction. At 0.22 strain, the basal pole intensity along the compression direction IP1 has formed, which is a clear sign of $\{10 \overline{1} 2\}\langle 10 \overline{1} 1\rangle$ deformation twinning. The calculated texture achieves very good agreement.

The single-load path tests in Fig. 1 were used to determine a single set of model parameters for the MS-EPSC model. In the simulations, we impose homogeneous boundary conditions for a uniaxial compression stress in the load direction and traction-free surfaces on the other orthogonal directions. As shown in Fig. 1 agreement with all monotonic tests was achieved. Table 1 summarizes the Burgers vector of all slip and twin modes established parameters. All subsequent calculations discussed hereinafter used these parameters and are predictions, not used as part of the fitting procedure.

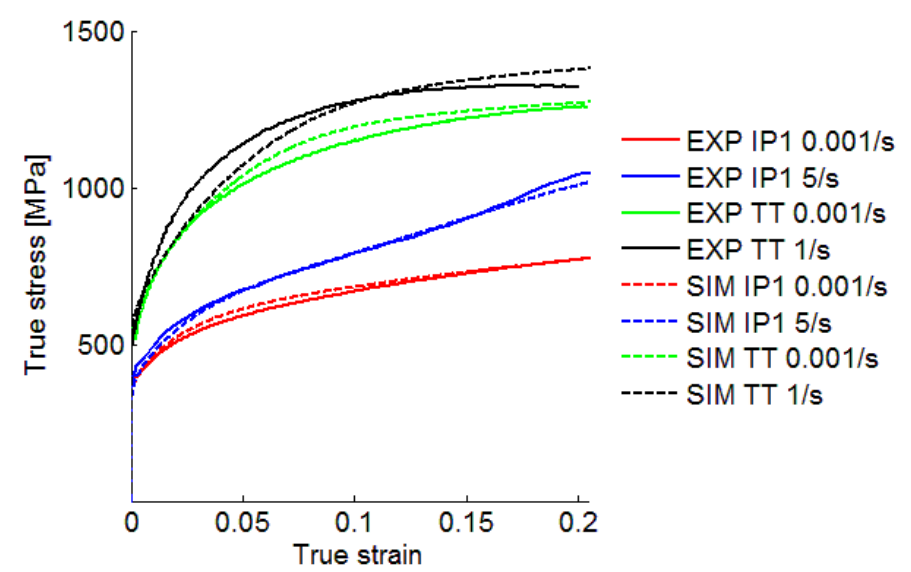

Figure 1 True stress-true strain response in compression at room temperature on annealed samples of rolled beryllium along the IP1 and TT direction under strain rates of $0.001 / \mathrm{s}, 1 / \mathrm{s}$ and 5/s: measured (solid lines) and predicted (dash-dot lines). 


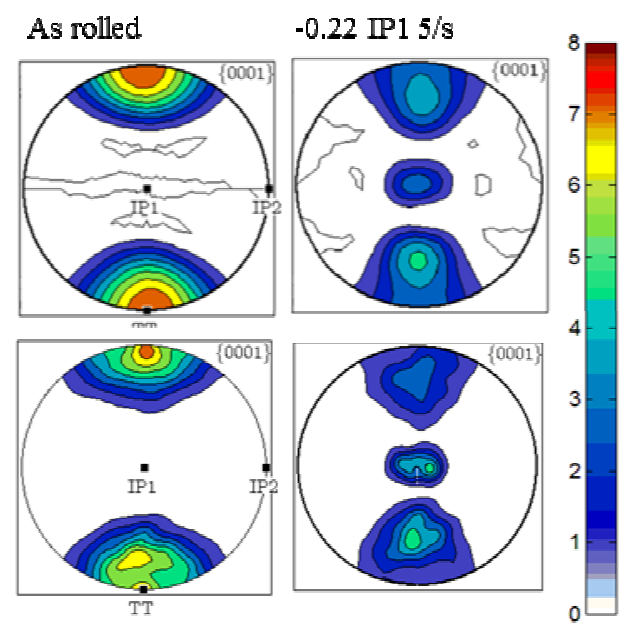

Figure 2 Basal pole figures showing the comparison between measured (top row) and simulated (bottom row) initial and deformed textured of rolled beryllium as indicated in the figure.

Table 1 Constitutive parameters for evolution of slip and twin system resistances.

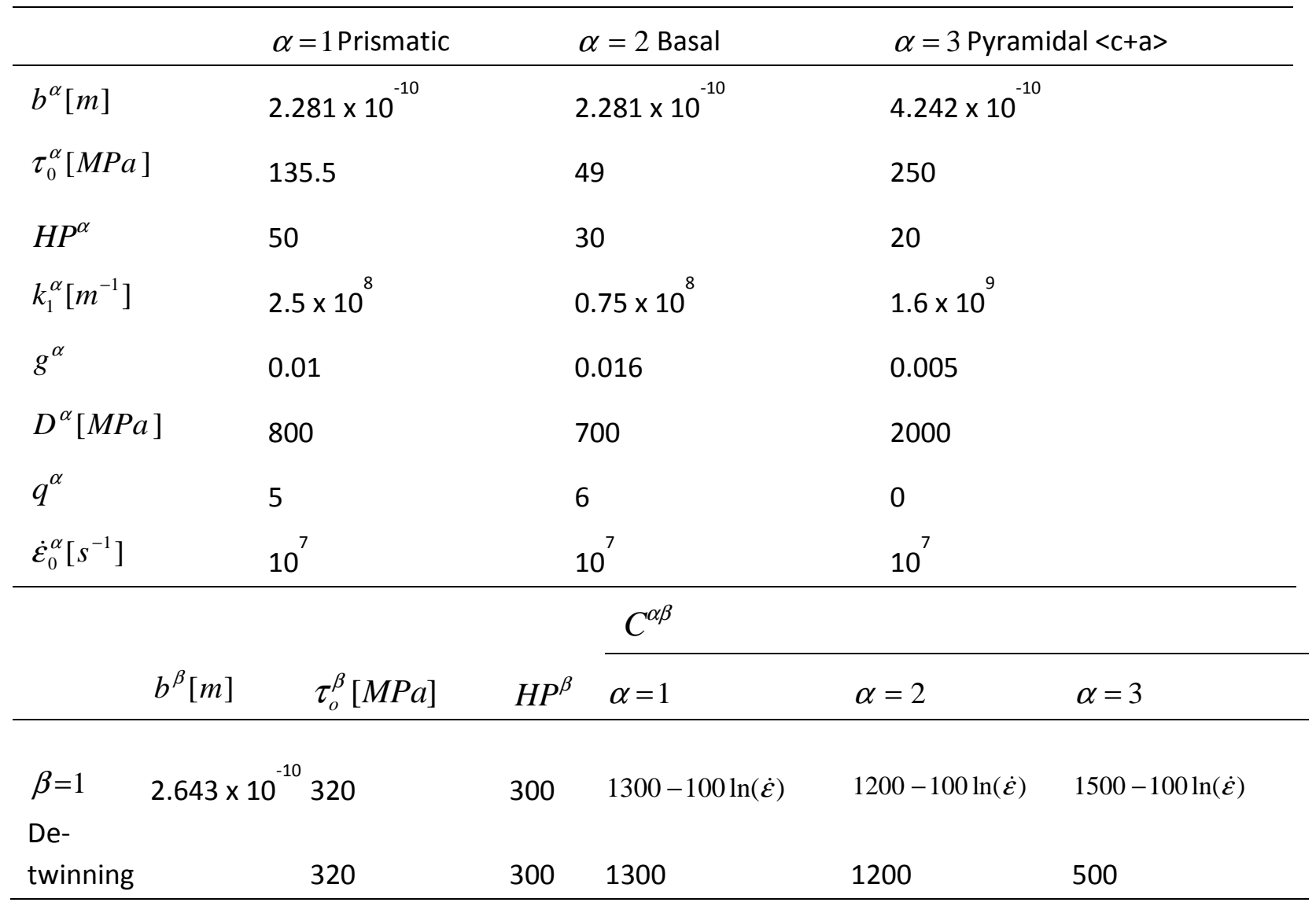


The initial slip resistance after including the Hall-Petch like term:

\begin{tabular}{lllll}
\hline & $\alpha=1$ & $\alpha=2$ & $\alpha=3$ & $\beta=1$ \\
\hline Initial CRSS $[\mathrm{MPa}]$ & 188.9 & 94.4 & 323 & 406.4 \\
\hline
\end{tabular}

\subsection{Model Predictions}

\subsection{Monotonic tests}

The predicted slip and twin activities associated with the stress-strain curves in Fig. 1 are given in Fig. 3. These reveal that among all the tests, only IP1 5/s is predicted to have substantial tensile twinning. Comparatively, in IP1 compression at the lower rate 0.001/s, less tensile twinning is predicted and in the two TT compression tests, no tensile twins are activated.

Experimental textures measured at different strain levels during the IP1 5/s and 0.001/s tests provide estimates of the twin volume fraction evolution, shown in Fig. 4 with dot symbols [63]. We observe that at the end of testing, the twin volume fraction in the 5/s test reached approximately 25\%, which is substantial. In Fig. 4, the predicted volume fractions of reoriented twin domains are compared with measurements. We see that the agreement is very good. Further, the model forecasts that within the tensile twin domains basal slip and pyramidal slip are active.

In the TT tests, in which twinning was not activated, we find that the deformation is instead accommodated via a mix of pyramidal and prismatic slip. These predictions of slip in the parent and twin domains are very similar to those reported in [68], which used the same dislocation density hardening law but within the VPSC framework. 

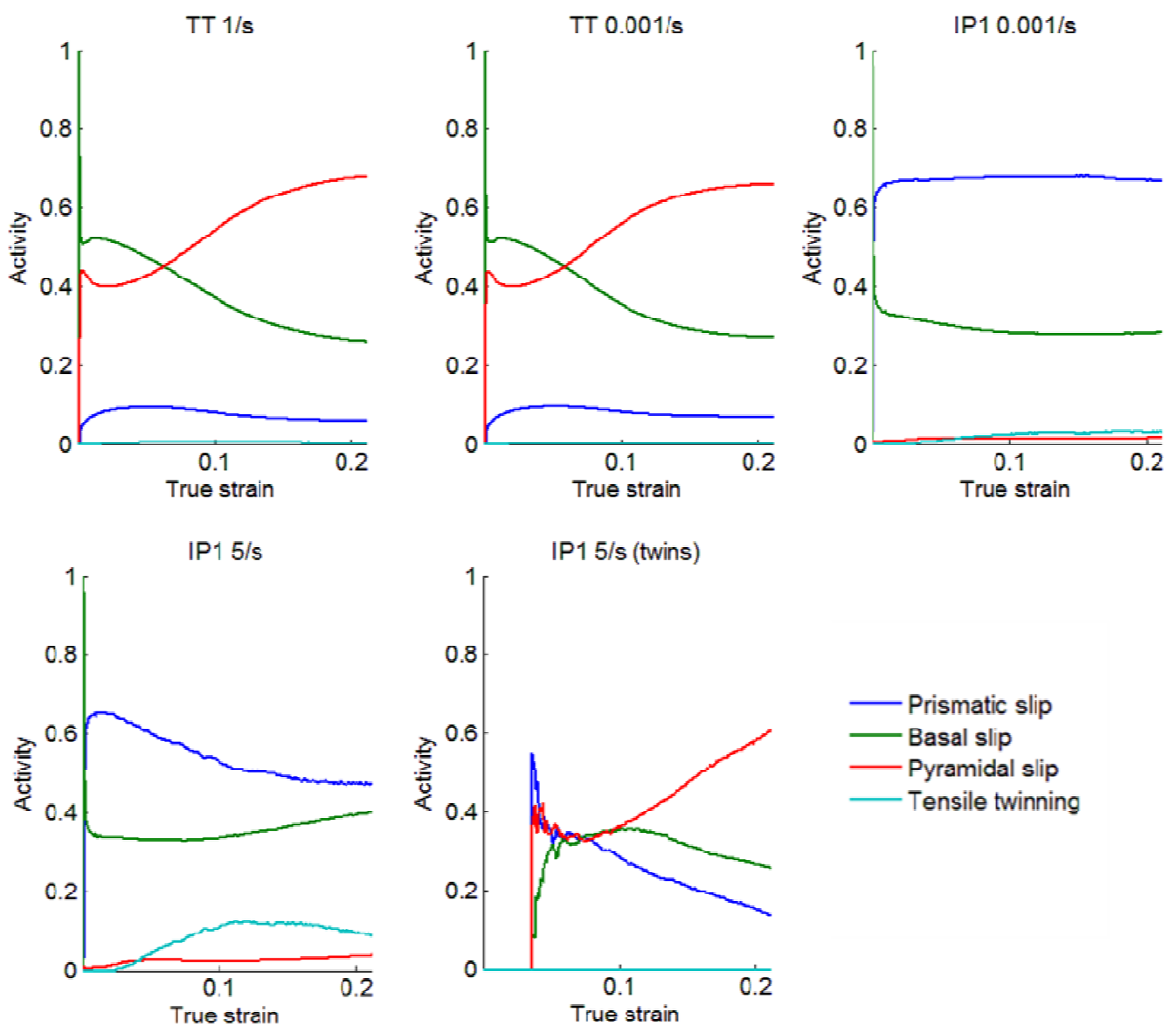

Figure 3 Predicted relative activities of each deformation mode contributing to plasticity in the parent domains for the compressed samples of beryllium in Fig. 1 and in the twin domains for the IP1 compression under 5/s.

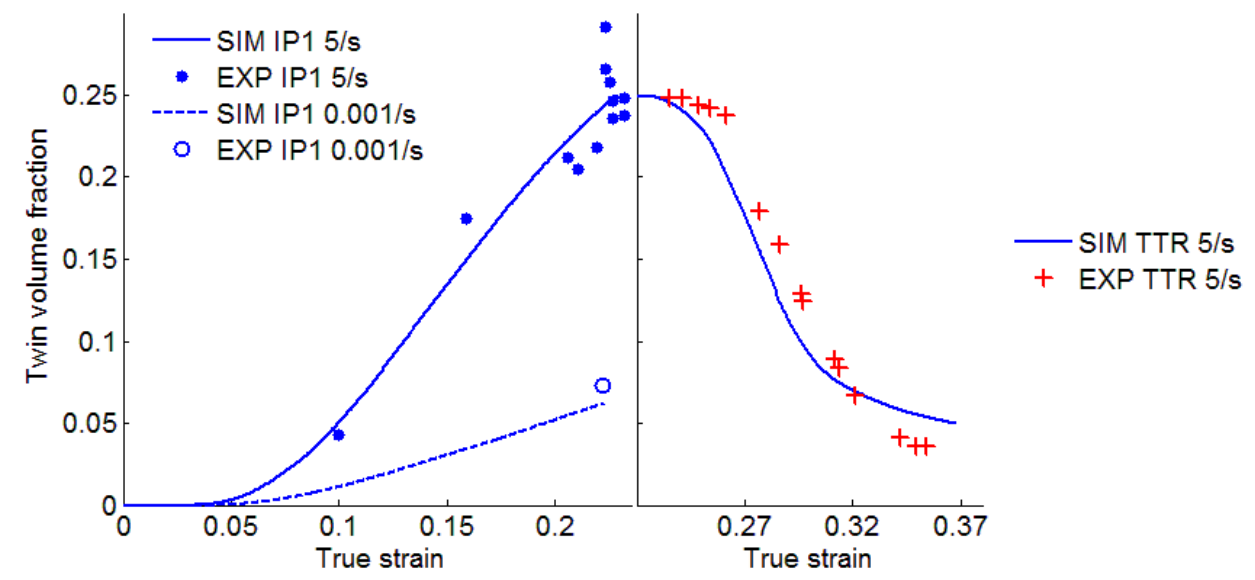


Figure 4 Evolution of the twin volume fraction during IP1 compression and secondary TTR compression under strain rates of $0.001 / \mathrm{s}$ and $5 / \mathrm{s}$ predicted by EPSC (lines) and measured by diffraction (symbols) as a function of strain. Measurements during the secondary path are based on in-situ integrated (0002) peak intensities.

\subsection{Strain path change tests}

To experimentally explore the effect of twinning on the deformation mechanisms activated in subsequent strain paths, the IP1 5/s samples were subjected either to through-thickness compression (TT) or orthogonal in plane direction (IP2). Each subsequent load test was carried out in one of two strain rates, the same rate of IP1 5/s or a much lower rate of $0.001 / \mathrm{s}$ [63].

Figure 5 shows the measured stress-strain response during the strain path sequences. Some features appear to be insensitive to the type of strain path and the strain rate. In all cases, the reload yield stress drops with respect to the stress reached at the end of the first path (IP1, 5/s). The hardening rate in the initial straining period of the second path, however, is significantly larger than that at the end of the first path. These changes have in prior works been attributed to backstresses and the flow of "polarized" or "reversible" dislocations [53-55, 57].

There are clearly other features of the stress-strain response that are dependent on strain path and strain rate, such as the flow stress and hardening rates during large strain deformation, beyond the first $5 \%$ of strain. These are related to the different deformation mechanisms activated. The IP1-TTR pathway represents a pseudo-reversal. Experimental evidence indicates de-twinning occurs in the TT reload [63]. First, in the TT-reload textures we see that as reloading proceeds, the intensity of the basal planes originally formed along the IP1 direction decreases. Also the twin volume fraction is observed to decrease (Fig. 4). The in-situ data indicate a delay in de-twinning. The (00.2) diffracted peak intensity measured during the in-situ TT crossreloading, which is representative of the twin volume fraction, indicates that the twin volume fraction is stable until -0.04 reloading plastic strain and then drops rapidly with increased plastic strain. The delay in the onset of de-twinning has been associated with the defect content in the microstructure during reloading [63]. The EPSC model successfully captures this effect, which we associate with accurate modeling of de-twin resistance and residual stresses. The IP1-IP2R pathway, in contrast, is a pseudo-cross-rolling strain path change. Texture analysis suggests that

new twins appear. As shown in Fig. 6, the concentration of basal poles along the IP2 has appeared after an additional 0.15 strain of IP2-reloading (5/s). Since the reload IP2R path 
changes the load state with respect to the grain orientations, these twins are different variants than those formed in the first path. The measured volume fraction of twins after the secondary deformation step of -0.15 plastic strain in the IP2R direction was 0.04 under $0.001 / \mathrm{s}$ and 0.13 under 5/s. The corresponding MS-EPSC predictions are 0.05 and 0.13 , respectively. Based on the experimental interpretation, modeling the constitutive response of these tests requires that the internal residual stresses, de-twinning, and multiple twin variants per grain are taken into account.

To calculate the constitutive response of these tests, we simulated 'continuously' the following three steps: cooling from the processing temperature of the material 850 to $300 \mathrm{~K}$ and subsequent deformation in the two-path deformation sequence by only changing the boundary conditions, temperature, and strain rate. In 'continuous' modeling, none of the microstructural variables, such as dislocation density, twin fraction, texture, and grain shapes, were reset in the subsequent deformation steps. The purpose of including the first cooling step was to estimate the initial state of thermal intergranular residual stresses $(d \boldsymbol{\sigma}=\mathbf{C}(d \boldsymbol{\varepsilon}-\boldsymbol{\alpha} d T))$, where $\boldsymbol{\alpha}$ is the thermal dilatation tensor that for Be is $\alpha_{11}=12.42 \times 10^{-6} \mathrm{~K}^{-1}, \alpha_{22}=12.42 \times 10^{-6} \mathrm{~K}^{-1}, \alpha_{33}=9.881 \times 10^{-6} \mathrm{~K}^{-1}$ and $\dot{T}$ is the temperature rate taken to be $80 \mathrm{~K}$ per increment (assumed to be uniform throughout the polycrystal). The calculated stress-strain curves and texture evolution in the subsequent paths are compared with the measurements in Figs. 5 and 6, respectively.

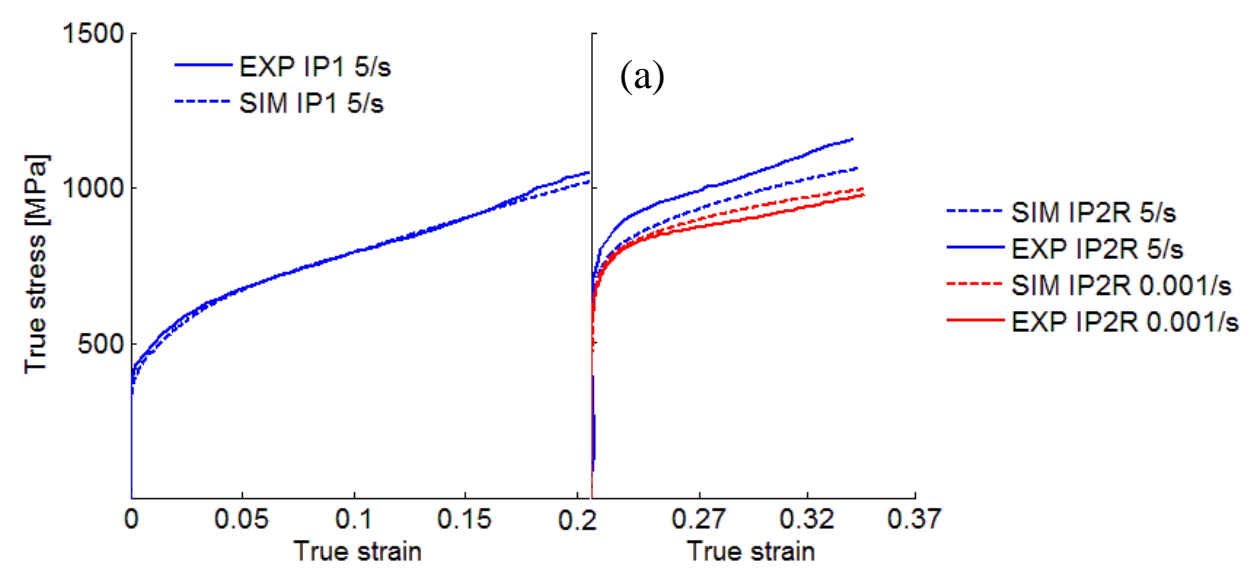




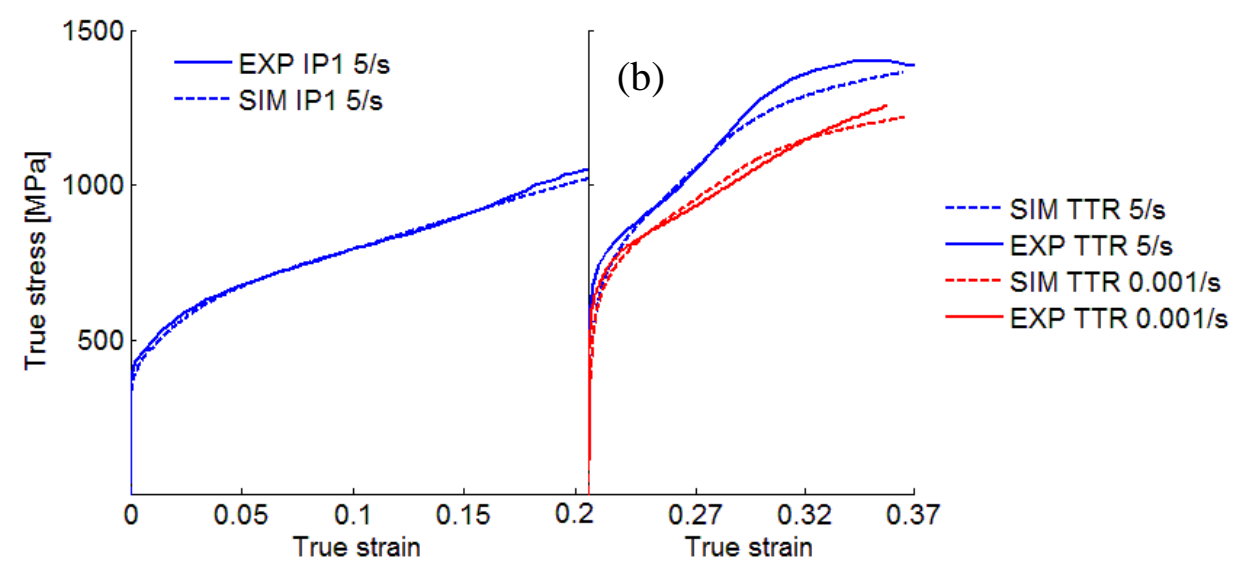

Figure 5 True stress-true strain response of rolled beryllium in primary IP1 compression under a strain rate of $5 / \mathrm{s}$ to a strain of -0.22 followed by (a) secondary IP2R compression and (b) secondary TTR compression under strain rates of $0.001 / \mathrm{s}$ and $5 / \mathrm{s}$ : measured (solid lines) and predicted (dash-dot lines).
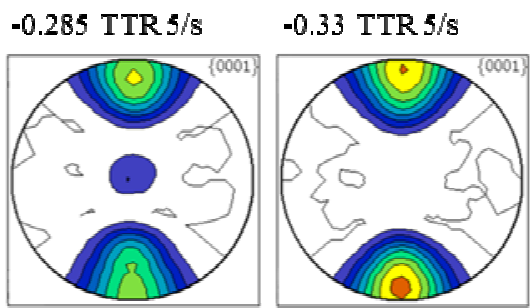

-0.37 TTR $5 / \mathrm{s}$
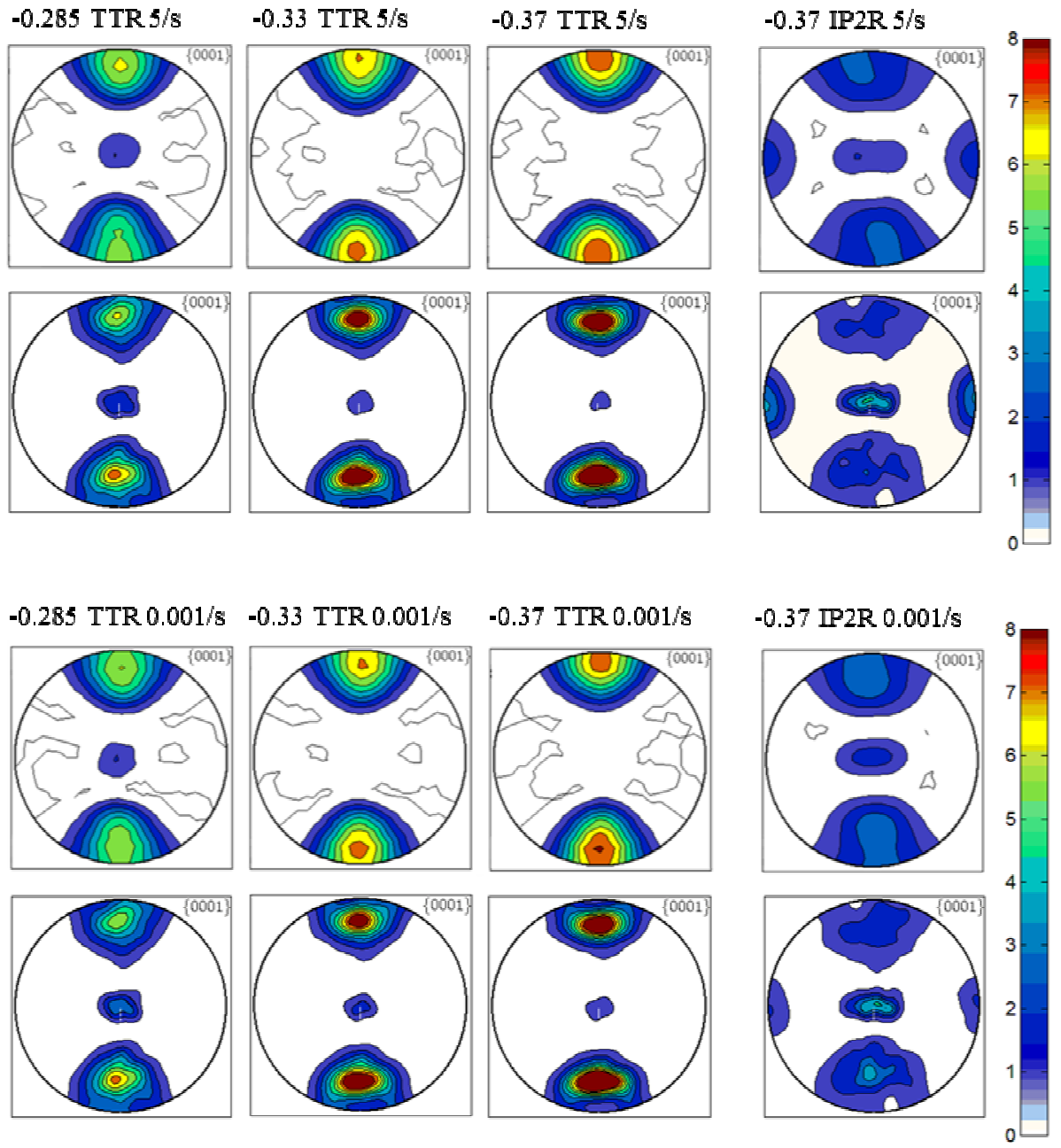
Figure 6 Basal pole figures showing measured (top row) and predicted (bottom row) texture evolution of rolled beryllium at various stages of deformation as indicated in the figure.

Figure 7 displays the predictions for average slip and twin activity in all the matrix grain regions (top row) and in all the twin domains formed during the first path (bottom row). As anticipated the IP1-TTR pathway leads to de-twinning, which is seen by the tensile twin activity of the same variant in the original twin domains. The new intensity of basal poles along the IP2 direction suggests that these are different twins than those formed in the primary IP1 5/s strain path.

In contrast, the model indicates that the IP1-IP2R pathway results in the nucleation of new twin variants. Figure 8 compares the calculated volume fraction of individual twin variants in the polycrystal after the primary path IP1 5/s and after the secondary path IP2R and TTR. While in TTR the same twin variants are decreasing in volume fraction, in IP2R the new twin volume variants are increasing in volume fraction. Figure 9 shows the percentage of grains with twins containing either one or multiple twin variants. Only a few grains were predicted to contain four variants and none contained all six variants through the strain-path-change deformation. 
(a)
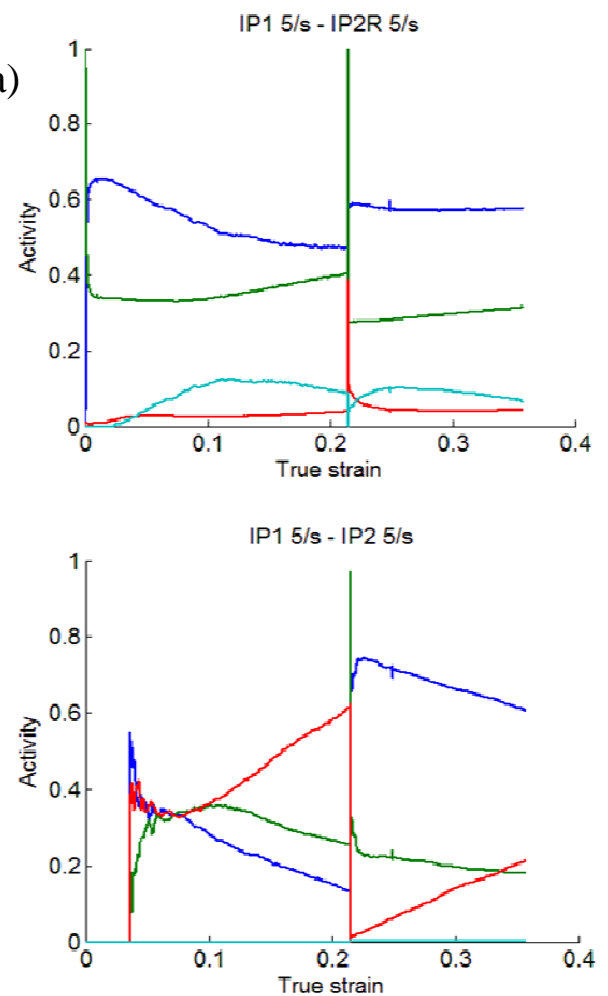

(b)
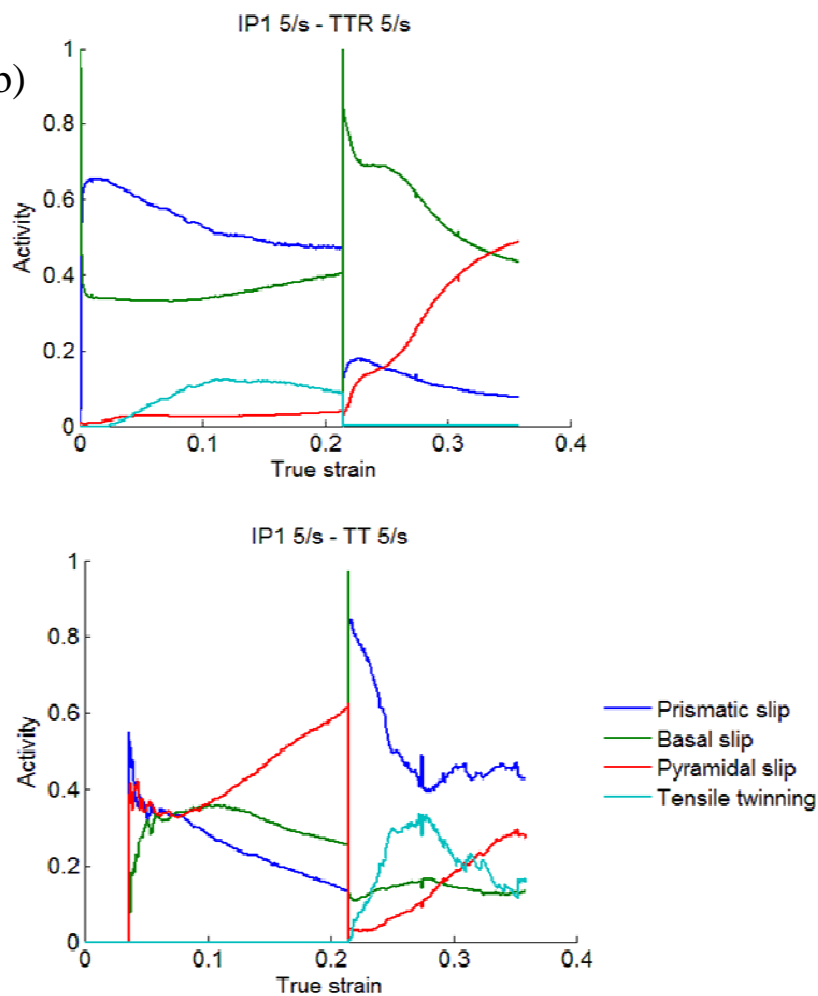

Figure 7 Predicted relative activities of the available deformation modes in the parent domains (top row) and twin domains (bottom row) under a strain rate of 5/s during (a) IP1 pre-straining followed by an IP2R compressive cross-reload and (b) IP1 pre-straining followed by a TTR compressive cross-reload. 


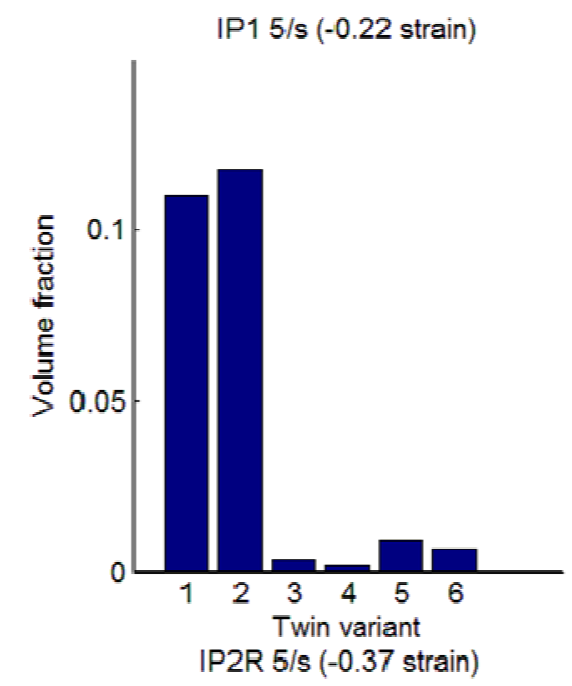

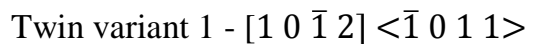
Twin variant $2-\left[\begin{array}{llllll}0 & 1 & \overline{1} & 2\end{array}\right]<0 \begin{array}{llll}1 & 1 & 1>\end{array}$ Twin variant $3-\left[\begin{array}{llll}\overline{1} & 1 & 0 & 2\end{array}\right]<\begin{array}{llll}1 & 1 & 0 & 1>\end{array}$

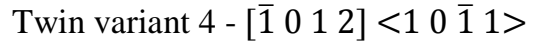
Twin variant $5-\left[\begin{array}{llll}0 & \overline{1} & 1 & 2\end{array}\right]<0 \begin{array}{llll}1 & \overline{1} & 1>\end{array}$ Twin variant $6-\left[\begin{array}{llll}1 & \overline{1} & 0 & 2\end{array}\right]<\overline{1} 1101>$
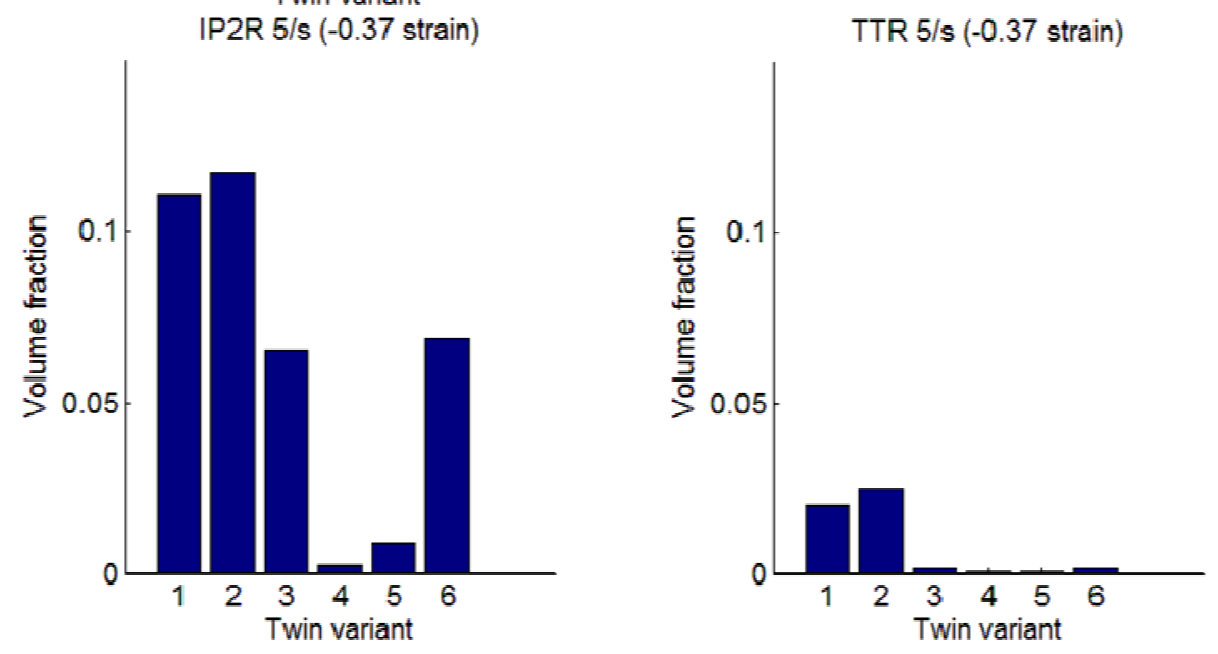

Figure 8 Volume fraction of active twin variants in the material at the end of primary IP1 and at the end of secondary IP2R and TTR, as indicated in the figure.

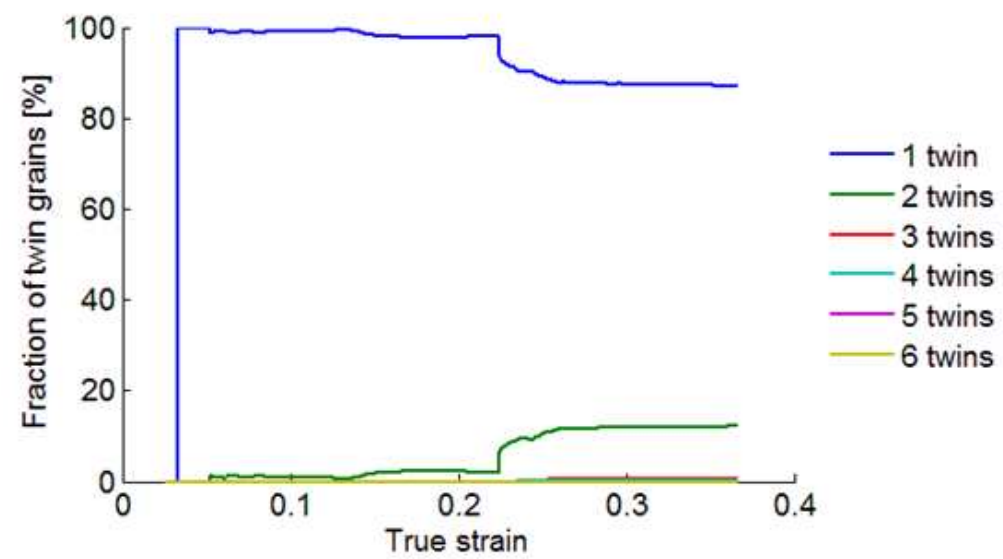

Figure 9 Fraction of parent grains with twins containing either one or multiple twin variants during IP1 5/s - IP2R 5/s. 


\subsection{Discussion}

\subsection{Initial drop}

From the data, we observe a drop in flow stress at the beginning of the reload path. In our earlier work using VPSC [57], we captured this drop by introducing reversible dislocations, and further assumed that a portion of these reversible dislocations are used during the reload while the remaining fraction is annihilated. Similarly and also in VPSC, [55] introduced reversible dislocations to capture the plateau in the reload curves and assumed that the previously generated reversible dislocations are annihilated during a reload.

In the current data, the initial drop arises regardless of the type of strain path change, whether a pseudo-reversal or pseudo-cross reload. Thus, it is not likely due to the glide of reversible dislocations since it is anticipated that this mechanism would be prevalent predominantly in the pseudo-reversal IP1-TTR pathway but not the pseudo-cross-loading IP1-IP2R pathway. This, however, does not imply that the flow of special 'directional' glide of dislocations (stress of type 3 ) is not occurring in the pseudo-reversal, only that it has a minimal effect on the initial flow stress during the reversal reload. We also observe and predict that the mechanisms carrying the deformation in each path are very different. Thus the initial drop in flow stress during reload is not dependent on whether new twins are nucleated or former twins grow or de-twin (shrink). Here we show that the MS-EPSC model was able to capture the flow stress changes starting from the initial state of the primary loading and to the end of the reloading path for all tests. Unlike prior attempts to model this data set, we account for elastic and plastic strain development. On this basis we conclude that the substantial drop in the stress and increase in hardening rate at the beginning of the second path predominantly result from the initial residual stresses developed in the first path.

\subsection{Lattice strains.}

One capability of the MS-EPSC model is the prediction of the evolution of elastic strains. As mentioned earlier, this evolution can be measured via in-situ diffraction techniques as the lattice strain experienced by certain crystallographic directions within a certain subset of diffracting grains. One important outcome when combining polycrystal modeling and lattice strain evolution is the evolution of strains in the matrix and twinned regions. Toward this end, Figure 10a compares measured and predicted strain evolution of the lattice strains in the $\{0002\},\{10 \overline{1} 0\}$, 
and $\{10 \overline{1} 1\}$ directions during the primary loading path IPC 5/s. Figure $10 \mathrm{~b}$ shows the volume fraction of the diffracting regions. For the present material, twinning causes the basal poles originally oriented along the TT to reorient along the IP1 and hence a signature of twin formation and growth is the increase in $\{0002\}$ diffracting volumes and lattice strains in $\{0002\}$ and a decrease in the $\{10 \overline{1} 0\}$ diffraction volumes as IP1 compression proceeds. Accordingly, the jump in the $\{0002\}$ curve seen in Fig. 10a corresponds to the onset of deformation twinning. The matrix regions associated with the grains that are twinning contribute to the $\{10 \overline{1} 0\}$ lattice strains. In response to twin growth, we observe that the lattice strains in the matrix regions become negative. Last, we remark that the model achieves good agreement with the data. On this note, we should mention that in simulation, the initial step of cooling from the processing temperature prior to deformation was included and was important for estimating the starting value of lattice strains in the material.

Good predictions of the residual lattice strains signify that the internal residual stresses are calculated accurately. With this in mind, we use the model to estimate the internal residual stresses. Figure 11 shows the volume distribution of internal residual stress in all three orthogonal sample directions at the end of the primary IP1 5/s loading. We find that the residual stresses are significant, ranging from -400 to $400 \mathrm{MPa}$, with a macroscopic average of zero, which is about $40 \%$ of the macroscopic stress achieved at the end of IP1 5/s deformation. The residual stress profiles upon IP1 preloading in the IP2 and TT directions influence the drop in the yield stress upon reloading.
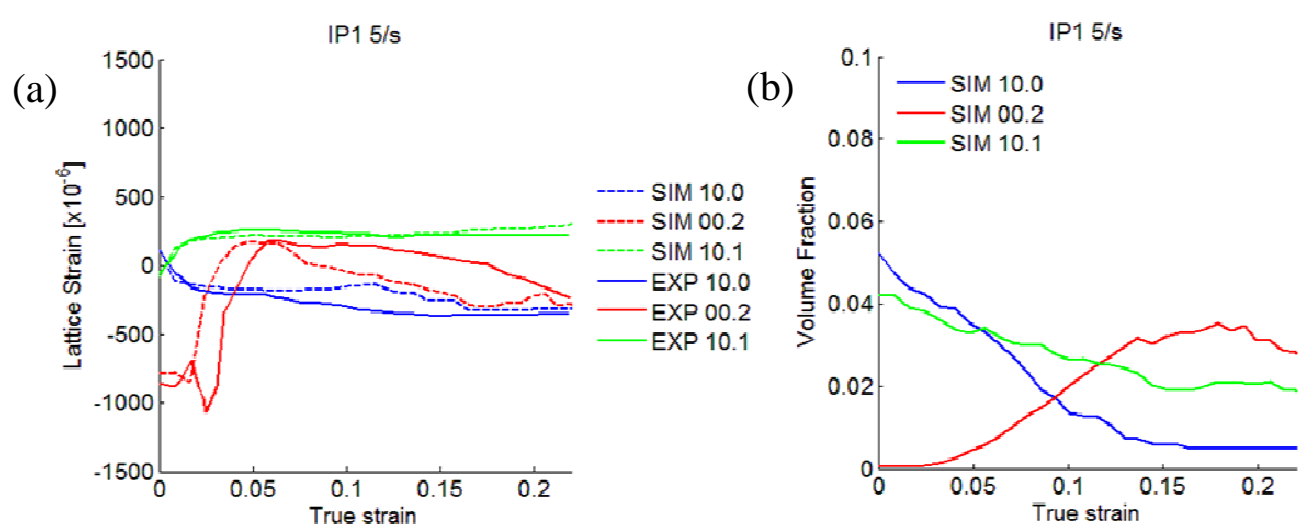

Figure 10 (a) Comparison of measured and predicted residual lattice strains during primary compression in the IP1 direction under strain rate of 5/s to a strain of -0.22 . (b) Volume fraction of grains contributing to the diffraction peaks. 

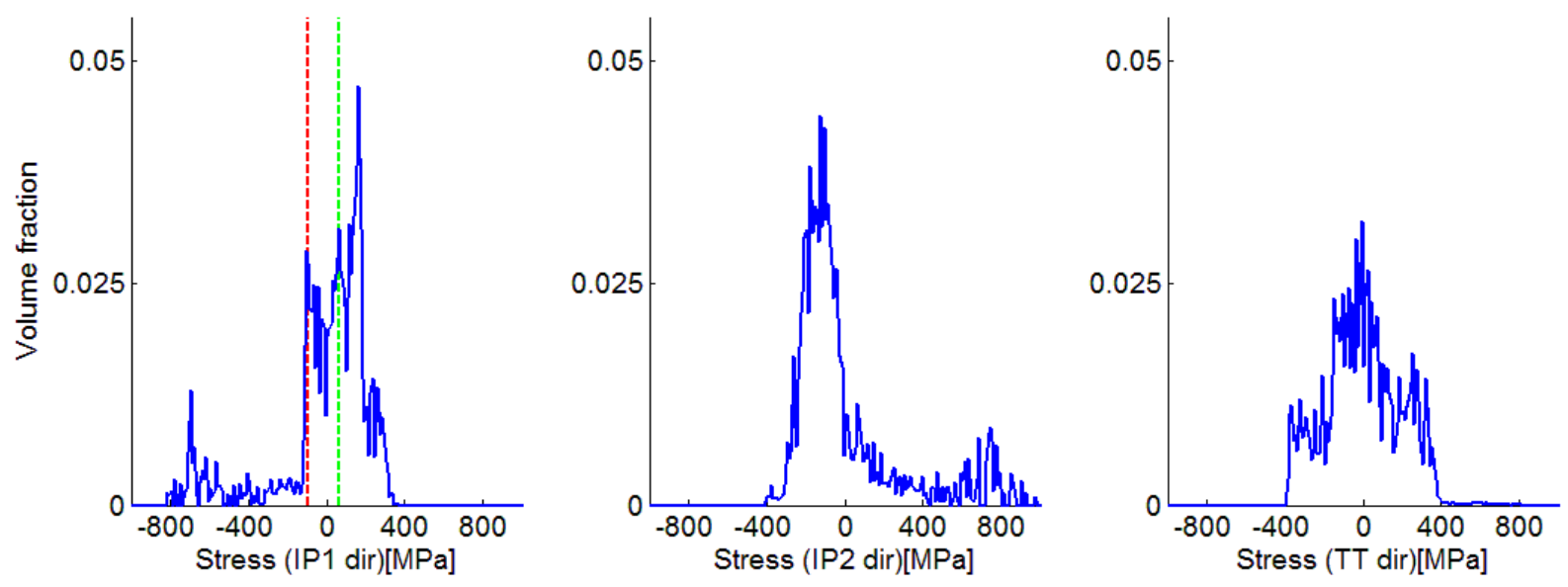

Figure 11 Distribution of residual stress at the end of primary IP1 loading ( 0.22 strain). The vertical lines in IP1 correspond to the average values of the residual stresses in the subsets of the diffracting grains (blue: (10.0), green: (10.1), red: (00.2)). Macroscopic average is zero.

\subsection{Stress fluctuations}

In the present simulations, stress fluctuations were introduced in the model for twin nucleation and impact the selection of twin variants formed in the model polycrystal during deformation. At the same time, lattice strain evolution is sensitive to the distribution of twin variants actually selected. Thus, in absence of direct microstructural evaluation by EBSD of the deformed material, we can employ lattice strain evolution data to provide an indication of the dispersion in twin variants formed. Figure 12 compares the simulation results without stress fluctuations with the measurements. The comparison suggests that stress fluctuations are important for obtaining agreement with the lattice strain evolution. Clearly unlike the predictions with stress fluctuations, the results in Fig. 12 do not achieve good agreement with the lattice strain evolution for the $\{10 \overline{1} 0\}$ lattice strains. With stress fluctuations, the volume fraction of grains giving rise to the $\{10 \overline{1} 0\}$ diffraction does not drop rapidly due to activation of different twin variants. In addition, with the stress fluctuations rate of twinning influences the $\{0002\}$ residual lattice strains curve to have lower rate of decrease with strain compared with no stress fluctuations.

The stress fluctuations can have several effects on variant selection. It can result in the formation of more than one twin variant per grain and formation of twin variants that have a relatively low Schmid factor and selection of twin variants of lower Schmid factor over those of 
higher Schmid factor. It can also cause twinning in grains not expected to twin, i.e., not favorably oriented for twinning. On this basis, we conclude from our model predictions of the lattice strains (with stress fluctuations) that the twin variants formed in the material do not necessarily correspond to those of the highest rank. In simulations without stress fluctuations, nearly all the twin variants formed in the model are rank 1 (with the highest value of Schmid factor). In simulations with stress fluctuations, this is not the case. Figure 8 compares the fraction of twin variants in the polycrystal at the end of primary path IP1 5/s and secondary path IP2. This conclusion is consistent with prior experimental studies on other HCP metals. Relatively large fractions of tensile twins not associated with the highest Schmid factor have been reported in deformed $\mathrm{Zr}$ and $\mathrm{Mg}$ using automated EBSD analyses of thousands of twins [21]. Our results here suggest that the same phenomenon of widely dispersed twin variant selection applies to $\{1012\}$ twinning in Be as well.
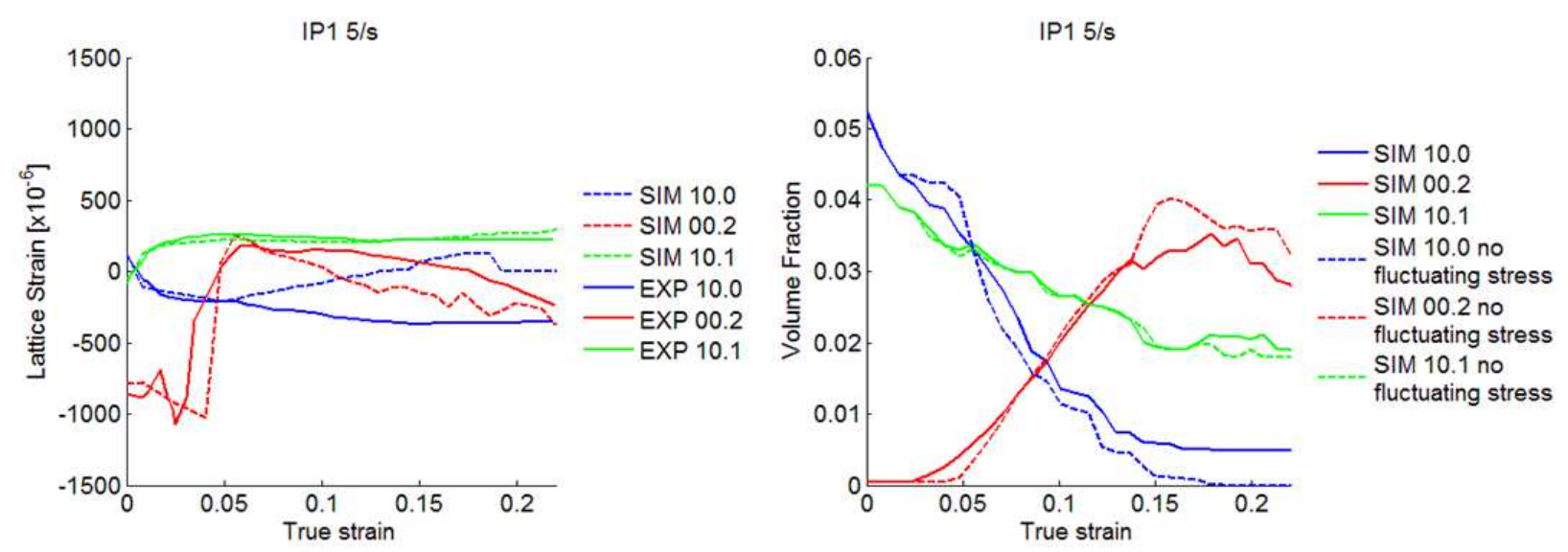

Figure 12 Predictions without fluctuating stresses. (a) Comparison of measured and predicted residual lattice strains during primary compression in the IP1 direction under a strain rate of 5/s to a strain of -0.22 . (b) Comparison of the volume fraction of grain oriented to give rise to the diffraction with and without fluctuation stresses.

\subsection{Conclusions}

We present a new and comprehensive polycrystal mean-field constitutive model based on the elastic-plastic self-consistent (EPSC) framework. In this model, referred to as multi-scale EPSC (MS-EPSC) we incorporate the most recent subgrain models for thermally activated glide, twin activation via statistical stress fluctuations, twin boundary hardening, and de-twinning. We apply the model to a set of strain path change tests on pure Be. The model is able to reproduce the 
transients in the stress-strain response, anisotropic hardening, and evolution of twin volume fraction and texture for all tests with a unique set of single-crystal hardening parameters. We find that the substantial changes in the initial flow stress behavior during the strain path change arise regardless of the cross-reloading direction. The drop in the stress and increase in hardening rate at the beginning of the second path predominantly result from the residual stresses developed in the first path. Accurate predictions of the residual lattice strains are indicative that the residual stresses are accurately calculated. Stress fluctuations have several effects on the $\{1012\}$ tensile twinning variant selection dispersing them in the sense that not all twins formed correspond to the variant with the highest Schmid factor. The implication of twin variant selection is seen in the improvements of the residual lattice strain predations.

The model presented here can be applied to other metals, deforming by multiple slip and twinning modes, deforming under a wide range of conditions, temperature, strain rate, and strain path conditions.

\section{Acknowledgements}

This work is based upon project supported by the National Science Foundation under grant CMMI-1301081. I.J.B. gratefully acknowledges support by a Laboratory Directed Research and Development grant ER20140348. C.T. acknowledges LANL's Advanced Simulation and Computing Program for support of this work.

\section{References}

[1] P.G. Partridge, Metallurgia Revised, 12 (1967) 169.

[2] M.H. Yoo, Metall. Mater. Trans. A, 12 (1981) 409-418.

[3] O. Cazacu, B. Plunkett, F. Barlat, International Journal of Plasticity, 22 (2006) 1171-1194.

[4] G.I. Taylor, Journal of the Institute of Metals, 62 (1938) 307-324.

[5] B.S. Fromm, B.L. Adams, S. Ahmadi, M. Knezevic, Acta Materialia, 57 (2009) 2339-2348.

[6] R.A. Lebensohn, C.N. Tomé, Acta Metallurgica et Materialia, 41 (1993) 2611-2624.

[7] P.A. Turner, C.N. Tomé, Acta Metallurgica et Materialia, 42 (1994) 4143-4153.

[8] S.R. Kalidindi, C.A. Bronkhorst, L. Anand, Journal of the Mechanics and Physics of Solids, 40 (1992) 537-569.

[9] F. Roters, P. Eisenlohr, L. Hantcherli, D.D. Tjahjanto, T.R. Bieler, D. Raabe, Acta Materialia, 58 (2010) 1152-1211.

[10] M. Knezevic, B. Drach, M. Ardeljan, I.J. Beyerlein, Computer Methods in Applied Mechanics and Engineering, 277 (2014) 239-259.

[11] B.L. Hansen, J.S. Carpenter, S.D. Sintay, C.A. Bronkhorst, R.J. McCabe, J.R. Mayeur, H.M. Mourad, I.J. Beyerlein, N.A. Mara, S.R. Chen, G.T. Gray Iii, International Journal of Plasticity, 49 (2013) 71-84. 
[12] R.A. Lebensohn, A.K. Kanjarla, P. Eisenlohr, International Journal of Plasticity, 32-33 (2012) 59-69.

[13] N. Jia, F. Roters, P. Eisenlohr, D. Raabe, X. Zhao, Acta Materialia, 61 (2013) 4591-4606.

[14] J. Mayeur, I. Beyerlein, C. Bronkhorst, H. Mourad, Materials, 7 (2014) 302-322.

[15] S.R. Kalidindi, A. Bhattacharya, R. Doherty, Proceedings of the Royal Society of London: Mathematical, Physical and Engineering Sciences., 460 (2004) 1935 - 1956

[16] P. Van Houtte, Textures and Microstructures, 8-9 (1988) 313-350.

[17] M. Knezevic, D.J. Savage, Computational Materials Science, 83 (2014) 101-106.

[18] M. Knezevic, S.R. Kalidindi, D. Fullwood, International Journal of Plasticity, 24 (2008) 1264-1276.

[19] M. Knezevic, H.F. Al-Harbi, S.R. Kalidindi, Acta Materialia, 57 (2009) 1777-1784.

[20] S.R. Niezgoda, A.K. Kanjarla, I.J. Beyerlein, C.N. Tomé, International Journal of Plasticity, 56 (2014) 119-138.

[21] I.J. Beyerlein, R.J. McCabe, C.N. Tome, International Journal for Multiscale Computational Engineering, 9 (2011) 459-480.

[22] I.J. Beyerlein, L.S. Tóth, C.N. Tomé, S. Suwas, Philosophical Magazine, 87 (2007) 885906.

[23] I.J. Beyerlein, N.A. Mara, D. Bhattacharyya, D.J. Alexander, C.T. Necker, International Journal of Plasticity, 27 (2011) 121-146.

[24] H. Abdolvand, M.R. Daymond, Journal of the Mechanics and Physics of Solids, 61 (2013) 803-818.

[25] A.J. Beaudoin, K.K. Mathur, P.R. Dawson, G.C. Johnson, International Journal of Plasticity, 9 (1993) 833-860.

[26] M. Knezevic, R.J. McCabe, R.A. Lebensohn, C.N. Tomé, C. Liu, M.L. Lovato, B. Mihaila, Journal of the Mechanics and Physics of Solids, 61 (2013) 2034-2046.

[27] M. Knezevic, R.J. McCabe, C.N. Tomé, R.A. Lebensohn, S.R. Chen, C.M. Cady, G.T. Gray Iii, B. Mihaila, International Journal of Plasticity, 43 (2013) 70-84.

[28] G.C. Kaschner, J.F. Bingert, C. Liu, M.L. Lovato, P.J. Maudlin, M.G. Stout, C.N. Tomé, Acta Materialia, 49 (2001) 3097-3108.

[29] J. Segurado, R.A. Lebensohn, J. Llorca, C.N. Tomé, International Journal of Plasticity, 28 (2012) 124-140.

[30] Balasubramanian, Computational Mechanics, 17. (1996) 209-225.

[31] D. Raabe, F. Roters, International Journal of Plasticity, 20 (2004) 339-361.

[32] A.J. Beaudoin, P.R. Dawson, K.K. Mathur, U.F. Kocks, D.A. Korzekwa, Computer Methods in Applied Mechanics and Engineering, 117 (1994) 49-70.

[33] M. Knezevic, M. Jahedi, Y.P. Korkolis, I.J. Beyerlein, Computational Materials Science, 95 (2014) 63-73.

[34] A. Kumar, P. Dawson, Computational mechanics, 17 (1995) 10-25.

[35] E. Voce, Journal of the Institute of Metals, 74 (1948) 537-562.

[36] S. Kok, A.J. Beaudoin, D.A. Tortorelli, International Journal of Plasticity, 18 (2002) 715741.

[37] P.S. Follansbee, U.F. Kocks, Acta Metallurgica, 36 (1988) 81-93.

[38] M. Knezevic, I.J. Beyerlein, T. Nizolek, N.A. Mara, T.M. Pollock, Materials Research Letters, 1 (2013) 133-140.

[39] I.J. Beyerlein, C.N. Tomé, International Journal of Plasticity, 24 (2008) 867-895. 
[40] M. Knezevic, L. Capolungo, C.N. Tomé, R.A. Lebensohn, D.J. Alexander, B. Mihaila, R.J. McCabe, Acta Materialia, 60 (2012) 702-715.

[41] N. Bertin, L. Capolungo, I.J. Beyerlein, International Journal of Plasticity, 49 (2013) 119144.

[42] J.W. Christian, S. Mahajan, Progress in Materials Science, 39 (1995) 1-157.

[43] I.J. Beyerlein, X. Zhang, A. Misra, Annual Review of Materials Research, 44 (2014) 329363.

[44] G.Y. Chin, W.L. Mamme, Transactions of the Metallurgical Society of AIME 245 (1969) 1211-1214.

[45] P. Van Houtte, Acta Metallurgica et Materialia, 26 (1978) 591-604.

[46] C.N. Tomé, R.A. Lebensohn, U.F. Kocks, Acta Metallurgica et Materialia, 39 (1991) 26672680.

[47] X. Wu, S.R. Kalidindi, C. Necker, A.A. Salem, Acta Materialia, 55 (2007) 423-432.

[48] S.R. Kalidindi, Journal of the Mechanics and Physics of Solids, 46 (1998) 267-271.

[49] G. Proust, C.N. Tomé, G.C. Kaschner, Acta Materialia, 55 (2007) 2137-2148.

[50] L. Capolungo, I.J. Beyerlein, G.C. Kaschner, C.N. Tomé, Materials Science and Engineering: A, 513-514 (2009) 42-51.

[51] G.C. Kaschner, C.N. Tomé, I.J. Beyerlein, S.C. Vogel, D.W. Brown, R.J. McCabe, Acta Materialia, 54 (2006) 2887-2896.

[52] I.J. Beyerlein, C.N. Tomé, Proceedings of the Royal Society A: Mathematical, Physical and Engineering Science, 466 (2010) 2517-2544.

[53] B. Peeters, M. Seefeldt, C. Teodosiu, S.R. Kalidindi, P. Van Houtte, E. Aernoudt, Acta Materialia, 49 (2001) 1607-1619.

[54] E. Rauch, J. Gracio, F. Barlat, G. Vincze, Modelling and Simulation in Materials Science and Engineering, 19 (2011) 1-18.

[55] K. Kitayama, C.N. Tomé, E.F. Rauch, J.J. Gracio, F. Barlat, International Journal of Plasticity, 46 (2013) 54-69.

[56] G. Proust, G.C. Kaschner, I.J. Beyerlein, B. Clausen, D.W. Brown, R.J. McCabe, C.N. Tomé, Experimental Mechanics, 50 (2010) 125-133.

[57] M. Knezevic, I.J. Beyerlein, D.W. Brown, T.A. Sisneros, C.N. Tomé, International Journal of Plasticity, 49 (2013) 185-198.

[58] I.J. Beyerlein, C.N. Tomé, International Journal of Plasticity, 23 (2007) 640-664.

[59] S. Mahesh, C.N. Tomé, R.J. McCabe, G.C. Kaschner, A. Misra, I.J. Beyerlein, Metallurgical and Materials Transactions A, 35 (2004) 3763-3774.

[60] J.A. Wollmershauser, B. Clausen, S.R. Agnew, International Journal of Fatigue, 36 (2012) 181-193.

[61] H. Wang, P.D. Wu, J. Wang, C.N. Tomé, International Journal of Plasticity, 49 (2013) 3652.

[62] B. Clausen, C.N. Tomé, D.W. Brown, S.R. Agnew, Acta Materialia, 56 (2008) 2456-2468.

[63] D.W. Brown, J.D. Almer, B. Clausen, P.L. Mosbrucker, T.A. Sisneros, S.C. Vogel, Materials Science and Engineering: A, 559 (2013) 29-39.

[64] J. Hutchinson, Proceedings of the Royal Society of London. A. Mathematical and Physical Sciences, 319 (1970) 247-272.

[65] C.J. Neil, J.A. Wollmershauser, B. Clausen, C.N. Tome, S.R. Agnew, International Journal of Plasticity, 26 (2010) 1772-1791.

[66] J.D. Eshelby, Proc R. Soc. Lond. A, 241 (1957) 376-396. 
[67] G. Proust, C.N. Tomé, A. Jain, S.R. Agnew, International Journal of Plasticity, 25 (2009) 861-880.

[68] D.W. Brown, I.J. Beyerlein, T.A. Sisneros, B. Clausen, C.N. Tomé, International Journal of Plasticity, 29 (2012) 120-135.

[69] A.L. Oppedal, H. El Kadiri, C.N. Tomé, G.C. Kaschner, S.C. Vogel, J.C. Baird, M.F. Horstemeyer, International Journal of Plasticity, 30-31 (2012) 41-61.

[70] I.J. Beyerlein, R.J. McCabe, C.N. Tomé, Journal of the Mechanics and Physics of Solids, 59 (2011) 988-1003.

[71] M. Knezevic, T. Nizolek, M. Ardeljan, I.J. Beyerlein, N.A. Mara, T.M. Pollock, International Journal of Plasticity, 57 (2014) 16-28.

[72] M. Ardeljan, I.J. Beyerlein, M. Knezevic, Journal of the Mechanics and Physics of Solids, 66 (2014) 16-31.

[73] M. Knezevic, J.S. Carpenter, M.L. Lovato, R.J. McCabe, Acta Materialia, 63 (2014) 162168.

[74] R. Madec, B. Devincre, L. Kubin, T. Hoc, D. Rodney, Science, 301 (2003) 1879-1882.

[75] U. Essmann, H. Mughrabi, Philosophical Magazine A, 40 (1979) 731-756.

[76] H. Mecking, U.F. Kocks, Acta Metallurgica et Materialia, 29 (1981) 1865-1875.

[77] U.F. Kocks, H. Mecking, Progress in Materials Science, 48 (2003) 171-273.

[78] M. Bourke, D. Dunand, E. Ustundag, Applied Physics A, 74 (2002) s1707-s1709.

[79] F. Aldinger, in: D. Webster, G.J. London (Eds.) Beryllium Science and Technology, New York, NY: Plenum Press, 1979, pp. 7.

[80] S. Jonsson, J. Beuers, Materials Science and Engineering, 91 (1987) 111-123.

[81] J.P. Poirier, J. Antolin, J.M. Dupouy, Canadian Journal of Physics, 45 (1967) 1221-1224.

[82] P.G. Partiridge, Metallurgia Revised, 12 (1967) 169.

[83] U.F. Kocks, C.N. Tome', H.-R. Wenk, Texture and Anisotropy, Cambridge University Press, Cambridge, 1998. 